\title{
Encapsulated high order difference operators on curvilinear non-conforming grids
}

Oskar Ålund and J an Nordström

The self-archived postprint version of this journal article is available at Linköping University Institutional Repository (DiVA):

http:// urn.kb.se/ resolve?urn=urn:nbn:se:liu:diva-154938

N.B.: When citing this work, cite the original publication.

Ålund, O., Nordström, J., (2019), Encapsulated high order difference operators on curvilinear nonconforming grids, J ournal of Computational Physics, 385, 209-224.

https:// doi.org/ 10.1016/j.jcp.2019.02.007

Original publication available at:

https:// doi.org/ 10.1016/j.jcp.2019.02.007

Copyright: Elsevier

http://www.elsevier.com/

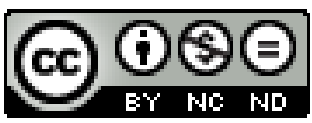




\title{
Encapsulated high order difference operators on curvilinear non-conforming grids
}

\author{
Oskar Ålund ${ }^{\mathrm{a}}$, Jan Nordström ${ }^{\mathrm{b}}$ \\ ${ }^{a}$ Department of Mathematics, Computational Mathematics, Linköping University, \\ SE-581 83 Linköping, Sweden (oskar.alund@liu.se). \\ ${ }^{b}$ Department of Mathematics, Computational Mathematics, Linköping University, \\ SE-581 83 Linköping, Sweden (jan.nordstrom@liu.se).
}

\begin{abstract}
Constructing stable difference schemes on complex geometries is an arduous task. Even fairly simple partial differential equations end up very convoluted in their discretized form, making them difficult to implement and manage. Spatial discretizations using so called summation-by-parts operators have mitigated this issue to some extent, particularly on rectangular domains, making it possible to formulate stable discretizations in a compact and understandable manner. However, the simplicity of these formulations is lost for curvilinear grids, where the standard procedure is to transform the grid to a rectangular one, and change the structure of the original equation.

In this paper we reinterpret the grid transformation as a transformation of the summation-by-parts operators. This results in operators acting directly on the curvilinear grid. Together with previous developments in the field of nonconforming grid couplings we can formulate simple, implementable, and provably stable schemes on general nonconforming curvilinear grids. The theory is applicable to methods on summation-by-parts form, including finite differences, discontinuous Galerkin spectral element, finite volume, and flux reconstruction methods. Time dependent advection-diffusion simulations corroborate the theoretical development.
\end{abstract}

Keywords: non-conforming grids, curvilinear mappings, weak interface couplings, summation-by-parts, stability, energy method 


\section{Introduction}

Two of the main characteristics of a powerful mathematical framework are encapsulation and reusability. The goal of this paper is to develop such a framework for discretizations of time dependent partial differential equations on curvilinear domains. For rectangular domains such a framework already exists in the form of Summation-By-Parts (SBP) operators. Combined with the notion of the Simultaneous Approximation Term (SAT) for imposing boundary conditions weakly [2, 17], it is possible to produce discretizations which closely resemble the continuous problem formulations. Furthermore, since SBP operators satisfy a discrete version of the integration by parts property (hence the name), the stability analysis of SBP-SAT discretizations also closely resembles the well-posedness analysis of the continuous problem. The SBP-SAT framework spans a large set of discretization methods, such as finite difference schemes (in the current paper), finite volume [9, 12], discontinuous Galerkin [4, and flux reconstruction methods [14].

However, when considering partial differential equations posed on curved domains, the similarities between the SBP-SAT discretization and the continuous formulation are to some extent lost. Because SBP operators typically act on rectangular grids (even though exceptions exist [3]), the standard procedure for dealing with curved grids has been to transform the equations to a rectangular domain through a change of variables (see e.g. [15, 16, 10, 13]). Note that such a transformation is not needed in order to derive energy estimates in the continuous setting - the transformation is done solely for discretization purposes. This procedure makes the discretization look unwieldy because it explicitly involves metric terms arising from the grid transforma-

tion. It also leads to unintuitive and convoluted stability analyses compared to the continuous setting.

Few approaches can be found in the literature that mitigate this issue. In [5] the authors achieve the convenience of being able to remain in untransformed space by constructing SBP operators directly on unstructured grids, which is a legitimate path but which inevitably leads to the computational inefficiencies typically associated with unstructured methods. Another key insight that can be used to recover the simplicity of SBP schemes on rectangular domains was presented in [7]. The authors were able to identify SBP operators approximating partial derivatives in physical space, in terms of standard SBP operators and metric coefficients. This is done on a fairly high level of abstraction however, and the details connecting the discrete and 
continuous theory are not clearly outlined.

In this paper we elaborate on the idea in [7] and reinterpret the grid transformation as a transformation of the SBP operators (we restrict ourselves to two dimensions for simplicity, but the idea is readily extended to three dimensions). Indeed, by defining appropriate numerical norms on the original domain and boundary, the grid transformation can be regarded as part of the construction of SBP operators with respect to these norms. Such operators act directly on the curved grid and lead to discretizations which mimic the continuous setting.

In terms of implementability this type of encapsulation is highly desirable. It allows us to write code for SBP operators acting on arbitrary curved grids. Similar to how there is no need construct the standard SBP operators from scratch for each new problem, there is no need to go through the entire grid transformation theory for each new problem on non-rectangular domains. Instead, the transformation theory is part of the operator implementation, rather than of the specific solver implementation. That is, once the operators have been implemented, the transformation step is eliminated from both code and theory. We simply supply a prewritten function with our curvilinear grid and it returns a curvilinear SBP operator ready for use. This leads to managable codes and easier debugging.

As an illustrative example of our encapsulation ambition we will apply the theory to nonconforming couplings of curvilinear grids (see Figure 4), where the notion of an intermediary so called glue grid, introduced in [6], is another key component with respect to encapsulation and reusability (note however that it is not straightforward to extend this coupling theory to three dimensions). The operators provided in [6] can be used with minor modification in order to construct stable couplings. Combined with the glue grid technique, the curvilinear SBP operators form a completely encapsulated and intuitive framework for discretizing partial differential equations on curved grids.

\section{The difference technique}

The difference schemes considered in this paper are constructed using two fundamental concepts: Summation-by-Parts (SBP) operators, and Simultaneous Approximation Terms (SATs). We give a brief introduction to these concepts here, for more details, see [2, 17].

Consider a uniform grid $x_{i}=i / N, i=0,1, \ldots, N$ on the unit interval. Let $P$ be a diagonal matrix approximating the standard $L^{2}$ inner product in the 


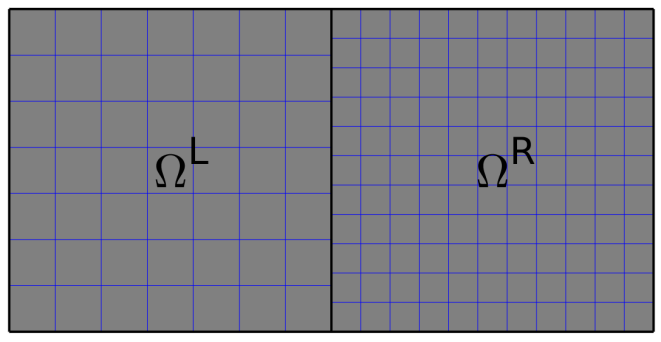

Figure 1: A non-conforming discretization of a rectangular domain $\Omega$.

sense that if $u, v \in L^{2}([0,1])$ are continuous functions and $\mathbf{u}, \mathbf{v}$ are vectors of $u$ and $v$ evaluated at the grid nodes, then $\langle\mathbf{u}, \mathbf{v}\rangle_{P}=\mathbf{u}^{\top} P \mathbf{v} \approx \int_{0}^{1} u v d x=$ $\langle u, v\rangle_{L^{2}}$. A matrix $D$ is called an $\mathrm{SBP}$ operator with respect to $P$ if for any $\mathbf{u}, \mathbf{v} \in \mathbb{R}^{N+1}$,

$$
\langle\mathbf{u}, D \mathbf{v}\rangle_{P}=u_{N} v_{N}-u_{0} v_{0}-\langle D \mathbf{u}, \mathbf{v}\rangle_{P},
$$

where $\mathbf{u}_{x} \approx D \mathbf{u}$ is vector of $u_{x}$ evaluated in the grid nodes. Note that (1) is a discrete version of the integration by parts property

$$
\left\langle u, v_{x}\right\rangle_{L^{2}}=u(1) v(1)-u(0) v(0)-\left\langle u_{x}, v\right\rangle_{L^{2}} .
$$

Discretely mimicking this behavior of the differentiation and integration operations is important because integration by parts is the central property used in deriving energy estimates for solutions to partial differential equations. Similarly, the summation-by-parts property is the central property used in deriving stability of SBP-SAT based discretizations.

\section{Two-dimensional SBP operators and non-conforming grids}

Let $\Omega=[-1,1] \times[0,1]$ and consider the subdomains $\Omega^{L}=[-1,0] \times[0,1]$ and $\Omega^{R}=[0,1] \times[0,1]$. We discretize the domain $\Omega$ by using equidistant but non-conforming grids on $\Omega^{L}$ and $\Omega^{R}$, see Figure 1 . More precisely we define the grid points $x_{i}^{L}=-1+i / N_{x}, i=0,1, \ldots, N_{x}$ and $y_{j}^{L}=j / N_{y}$, $j=0,1, \ldots, N_{y}$ associated to $\Omega^{L}$, and $x_{i}^{R}=i / M_{x}, i=0,1, \ldots, M_{x}$ and $y_{j}^{R}=j / M_{y}, j=0,1, \ldots, M_{y}$ associated to $\Omega^{R}$. To the left subdomain $\Omega^{L}$ we associate SBP operators $D_{x}, D_{y}$ in the $x$ - and $y$-directions together with corresponding diagonal norms $P_{x}$ and $P_{y}$. The Kronecker product $\mathbf{P}=P_{x} \otimes$ $P_{y}$ defines an inner product on the space $\mathbb{R}^{\left(N_{x}+1\right)\left(N_{y}+1\right)}$ approximating the 
standard inner product in $L^{2}\left(\Omega^{L}\right)$. This means that if $u, v \in L^{2}\left(\Omega^{L}\right)$, and $\mathbf{u}, \mathbf{v}$ are vectors formed by evaluating $u$ and $v$ at the grid points, then

$$
\langle\mathbf{u}, \mathbf{v}\rangle_{\mathbf{P}}=\mathbf{u}^{\top} \mathbf{P} \mathbf{v} \approx\langle u, v\rangle_{L^{2}}=\iint_{\Omega^{L}} u v d x d y .
$$

Furthermore, two-dimensional (2D) SBP operators associated to the inner product $\mathbf{P}$ can be constructed by forming the Kronecker products $\mathbf{D}_{x}=$ $D_{x} \otimes I_{y}$ and $\mathbf{D}_{y}=I_{x} \otimes D_{y}$, where $I_{x}$ and $I_{y}$ are identity matrices of size $N_{x}+1$ and $N_{y}+1$ respectively. The SBP property is inherited in the sense that

$$
\begin{aligned}
& \left\langle\mathbf{u}, \mathbf{D}_{x} \mathbf{v}\right\rangle_{\mathbf{P}}=\langle\mathbf{u}, \mathbf{v}\rangle_{\mathbf{P}_{e}}-\langle\mathbf{u}, \mathbf{v}\rangle_{\mathbf{P}_{w}}-\left\langle\mathbf{D}_{x} \mathbf{u}, \mathbf{v}\right\rangle_{\mathbf{P}} \\
& \left\langle\mathbf{u}, \mathbf{D}_{y} \mathbf{v}\right\rangle_{\mathbf{P}}=\langle\mathbf{u}, \mathbf{v}\rangle_{\mathbf{P}_{n}}-\langle\mathbf{u}, \mathbf{v}\rangle_{\mathbf{P}_{s}}-\left\langle\mathbf{D}_{y} \mathbf{u}, \mathbf{v}\right\rangle_{\mathbf{P}}
\end{aligned}
$$

where $\mathbf{P}_{e}, \mathbf{P}_{w}, \mathbf{P}_{n}, \mathbf{P}_{s}$ induce inner products on the boundaries and are defined as

$$
\mathbf{P}_{e}=E_{N_{x}} \otimes P_{y}, \quad \mathbf{P}_{w}=E_{0} \otimes P_{y}, \quad \mathbf{P}_{n}=P_{x} \otimes E_{N_{y}}, \quad \mathbf{P}_{s}=P_{x} \otimes E_{0} .
$$

Here $E_{i}$ denotes a matrix with a single 1 at position $(i, i)$ and zeros elsewhere.

Similarly, to the right subdomain $\Omega^{R}$ we associate SBP operators $D_{x}$, $D_{y}$ in the $x$ - and $y$-directions together with corresponding diagonal norms $H_{x}$ and $H_{y}$. Note that while we reuse the notation $D_{x}$ and $D_{y}$ it will be clear from the context which operator is being used. Corresponding 2D SBP operators $\mathbf{D}_{x}, \mathbf{D}_{y}$, and inner products matrices $\mathbf{H}, \mathbf{H}_{e}, \mathbf{H}_{w}, \mathbf{H}_{s}, \mathbf{H}_{n}$ are defined analogously.

Finally we define the discrete gradient and Laplacian,

$$
\nabla \mathbf{u}=\left(\mathbf{D}_{x} \mathbf{u}, \mathbf{D}_{y} \mathbf{u}\right), \quad \Delta \mathbf{u}=\mathbf{D}_{x}^{2} \mathbf{u}+\mathbf{D}_{y}^{2} \mathbf{u}
$$

Note that $\nabla \mathbf{u}$ is a pair of vectors. It is a notational device allowing us to formulate discrete expressions that mimic the continuous form. For example, if $\mathbf{a}=(a, b)$ is a velocity vector and $u$ is a function approximated by $\mathbf{u}$, then $\mathbf{a} \cdot \nabla \mathbf{u}:=a \mathbf{D}_{x} \mathbf{u}+b \mathbf{D}_{y} \mathbf{u}$ approximates $\mathbf{a} \cdot \nabla u$ in the grid nodes.

\section{Curvilinear SBP operators}

To be able to handle non-rectangular domains $\Omega$ it is convenient to define SBP operators acting directly on curvilinear grids. This would make it 


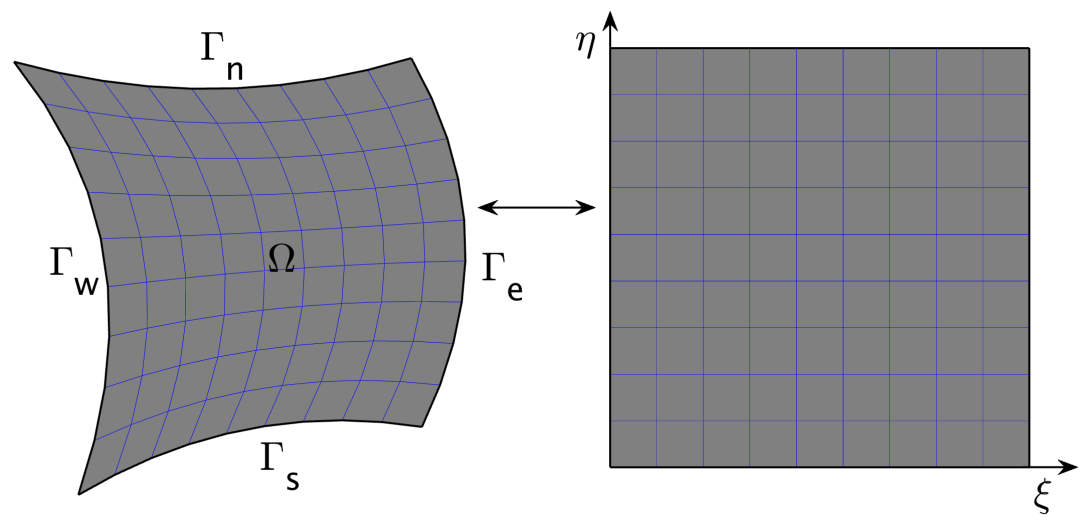

Figure 2: A smooth transformation from curvilinear to square grid.

possible to formulate discrete schemes directly on curved domains with the same simplicity as in the rectangular case. Our ambition is to construct a procedure where the traditional technique of transforming curved domains to rectangular domains is an effort that need only be considered once in order to construct appropriate SBP operators. Once these operators have been constructed, there is no need for coordinate transforms in the involved equations, and the stability analysis will perfectly mimic the continuous energy method in the untransformed space.

Remark 1. Note that the schemes formulated using these operators will be numerically equivalent to traditional schemes. The encapsulation procedure is entirely a notational device meant to increase readability and intuition (and hence reduce mistakes) during the construction and implementation of curvilinear schemes. That is, the computational efficiency is unaffected when compared to traditional formulations.

Consider the curved domain $\Omega$ along with its curvilinear grid $\Omega_{h}$ shown in Figure 2 and let $\Gamma=\partial \Omega$. The construction procedure is split into three parts:

- Construct a quadrature matrix $\mathbf{P}$ which approximates the standard inner product in $L^{2}(\Omega)$.

- Construct boundary quadrature matrices $\mathbf{P}_{w}, \mathbf{P}_{e}, \mathbf{P}_{s}, \mathbf{P}_{n}$ which approximate the inner products in $L^{2}\left(\Gamma_{w}\right), L^{2}\left(\Gamma_{e}\right), L^{2}\left(\Gamma_{s}\right), L^{2}\left(\Gamma_{n}\right)$. 
- Construct SBP operators $\mathbf{D}_{x}$ and $\mathbf{D}_{y}$ which mimic the integration by parts formulas $\left\langle u, v_{x}\right\rangle=\left\langle u, v n_{x}\right\rangle_{L^{2}(\Gamma)}-\left\langle u_{x}, v\right\rangle_{L^{2}(\Omega)}$ and $\left\langle u, v_{y}\right\rangle=$ $\left\langle u, v n_{y}\right\rangle_{L^{2}(\Gamma)}-\left\langle u_{y}, v\right\rangle_{L^{2}(\Omega)}$.

The curvilinear grid is made up of the grid points $\Omega_{h}=\left\{\left(x_{i j}, y_{i j}\right): i=\right.$ $0,1, \ldots, M, j=0,1, \ldots, N\}$, while the square reference grid is made up of the grid points $\left(\xi_{i}, \eta_{j}\right), \xi_{i}=i / M, i=0,1, \ldots, M, \eta_{j}=j / N, j=0,1, \ldots, N$. We assume that there is a smooth, bijective, orientation preserving transformation (i.e. with a strictly positive Jacobian) $\Omega \rightarrow[0,1]^{2},(x, y) \mapsto(\xi, \eta)$, mapping the grid points $\left(x_{i j}, y_{i j}\right)$ in the curvilinear grid to corresponding grid points $\left(\xi_{i}, \eta_{j}\right)$ in the square grid.

We begin by defining an appropriate quadrature matrix $\mathbf{P}$ to be used as a discrete version of the standard inner product in $L^{2}(\Omega)$. Recall that for a given function $u \in L^{2}(\Omega)$ we have

$$
\int_{\Omega} u(x, y) d x d y=\int_{[0,1]^{2}} J(\xi, \eta) u(x(\xi, \eta), y(\xi, \eta)) d \xi d \eta
$$

where $J(\xi, \eta)=\operatorname{det}\left(\frac{\partial(x, y)}{\partial(\xi, \eta)}\right)=x_{\xi} y_{\eta}-x_{\eta} y_{\xi}$.

The coordinate transformation is typically not exlpicitly known since the curvilinear grid is constructed directly with respect to the curved domain. Hence the partial derivatives of $x$ and $y$ with respect to $\xi$ and $\eta$ must be approximated. To do this we let $D_{\xi}$ and $D_{\eta}$ be SBP operators with associated diagonal quadrature matrices $P_{\xi}$ and $P_{\eta}$ in the $\xi$ - and $\eta$-directions using the grid points $\xi_{i}$ and $\eta_{j}$. Then $\mathbf{D}_{\xi}=D_{\xi} \otimes I_{\eta}$ and $\mathbf{D}_{\eta}=I_{\xi} \otimes D_{\eta}$ are twodimensional SBP operators with respect to the quadrature matrix $\mathbf{P}_{\xi, \eta}=$ $P_{\xi} \otimes P_{\eta}$

Now let $\mathbf{x}$ and $\mathbf{y}$ be vectors containing the grid points $x_{i j}$ and $y_{i j}$ respectively. We define the discrete partial derivatives of $x$ and $y$,

$\mathbf{X}_{\xi}=\operatorname{diag}\left(\mathbf{D}_{\xi} \mathbf{x}\right), \quad \mathbf{X}_{\eta}=\operatorname{diag}\left(\mathbf{D}_{\eta} \mathbf{x}\right), \quad \mathbf{Y}_{\xi}=\operatorname{diag}\left(\mathbf{D}_{\xi} \mathbf{y}\right), \quad \mathbf{Y}_{\eta}=\operatorname{diag}\left(\mathbf{D}_{\eta} \mathbf{y}\right)$,

along with the discrete Jacobian

$$
\mathbf{J}=\mathbf{X}_{\xi} \mathbf{Y}_{\eta}-\mathbf{X}_{\eta} \mathbf{Y}_{\xi}
$$

Using $\mathbf{J}$ and $\mathbf{P}_{\xi, \eta}$ we can now define a quadrature matrix $\mathbf{P}$ on the curvilinear grid $\left(x_{i j}, y_{i j}\right)$. 
Definition 1. Given a curved, quadrilateral domain $\Omega$ and an associated curvilinear grid $\Omega_{h}=\left\{\left(x_{i j}, y_{i j}\right)\right\}$, the matrix $\mathbf{P}=\mathbf{P}_{\xi, \eta} \mathbf{J}$ is called an $S B P$ quadrature on $\Omega_{h}$.

An SBP quadrature $\mathbf{P}$ on $\Omega_{h}$ acts as a discrete integration operator and inner product on the whole domain. If $u, v \in L^{2}(\Omega)$ and $\mathbf{u}, \mathbf{v}$ are vectors with $u$ and $v$ evaluated on $\Omega_{h}$, then

$$
\langle\mathbf{u}, \mathbf{v}\rangle_{\mathbf{P}}:=\mathbf{u}^{\top} \mathbf{P} \mathbf{v} \approx\langle u, v\rangle_{L^{2}(\Omega)}=\int_{\Omega} u v d x d y .
$$

Next we construct boundary quadrature matrices $\mathbf{P}_{k}$ such that $\langle\mathbf{u}, \mathbf{v}\rangle_{\mathbf{P}_{k}} \approx$ $\langle u, v\rangle_{L^{2}\left(\Gamma_{k}\right)}$ for $k \in\{w, e, s, n\}$. Recall that a curve integral, given a parametrization of the curve, can be written as a standard integral over the parameter domain. For example, $\Gamma_{w}$ can be parametrized by the function

$$
\eta \mapsto(x(0, \eta), y(0, \eta)), \eta \in[0,1] .
$$

It follows that

$$
\int_{\Gamma_{w}} u d s=\int_{0}^{1} u(x(0, \eta), y(0, \eta)) \sqrt{x_{\eta}^{2}(0, \eta)+y_{\eta}^{2}(0, \eta)} d \eta .
$$

Hence, the boundary integral $\int_{\Gamma_{w}} u d s$ can be approximated using the boundary quadrature

$$
\left(E_{0} \otimes P_{\eta}\right) \sqrt{\mathbf{X}_{\eta}^{2}+\mathbf{Y}_{\eta}^{2}} .
$$

This is simply a diagonal matrix containing the segment lengths of the western boundary. Similar expressions for the remaining boundaries are summarized in the following definition.

Definition 2. Let $\Omega$ be a curved, quadrilateral domain with boundary $\Gamma$ and an associated curvilinear grid $\Omega_{h}=\left\{\left(x_{i j}, y_{i j}\right)\right\}$. The matrices

$$
\begin{aligned}
\mathbf{P}_{w} & =\left(E_{0} \otimes P_{\eta}\right)\left(\mathbf{X}_{\eta}^{2}+\mathbf{Y}_{\eta}^{2}\right)^{\frac{1}{2}} \\
\mathbf{P}_{e} & =\left(E_{M} \otimes P_{\eta}\right)\left(\mathbf{X}_{\eta}^{2}+\mathbf{Y}_{\eta}^{2}\right)^{\frac{1}{2}} \\
\mathbf{P}_{s} & =\left(P_{\xi} \otimes E_{0}\right)\left(\mathbf{X}_{\xi}^{2}+\mathbf{Y}_{\xi}^{2}\right)^{\frac{1}{2}} \\
\mathbf{P}_{n} & =\left(P_{\xi} \otimes E_{N}\right)\left(\mathbf{X}_{\xi}^{2}+\mathbf{Y}_{\xi}^{2}\right)^{\frac{1}{2}}
\end{aligned}
$$

are called SBP boundary quadratures on $\Omega_{h}$. 
An SBP boundary quadrature on $\Omega_{h}$ acts as a discrete integration operator and inner product on the boundary. If $u, v \in L^{2}(\Omega)$ and $\mathbf{u}, \mathbf{v}$ are vectors with $u$ and $v$ evaluated on $\Omega_{h}$, then

$$
\langle\mathbf{u}, \mathbf{v}\rangle_{\mathbf{P}_{k}}:=\mathbf{u}^{\top} \mathbf{P}_{k} \mathbf{v} \approx\langle u, v\rangle_{L^{2}\left(\Gamma_{k}\right)}=\oint_{\Gamma_{k}} u v d s \text { for } k \in\{w, e, s, n\} .
$$

Next we construct SBP operators $\mathbf{D}_{x}$ and $\mathbf{D}_{y}$ on $\Omega_{h}$. The goal is to construct these operators so that they mimic the continuous integration by parts property. Recall that if $u, v \in L^{2}(\Omega)$ are differentiable functions and $\left(n_{x}, n_{y}\right)$ is the outward pointing unit normal, then

$$
\begin{aligned}
\int_{\Omega} u v_{x} d x d y & =\oint_{\Gamma} u v n_{x} d s-\int_{\Omega} u_{x} v d x d y \\
& =\sum_{k=w, e, s, n} \oint_{\Gamma_{k}} u v n_{x} d s-\int_{\Omega} u_{x} v d x d y
\end{aligned}
$$

Or, on inner product form:

$$
\left\langle u, v_{x}\right\rangle_{L^{2}(\Omega)}=\sum_{k=w, e, s, n}\left\langle u, n_{x} v\right\rangle_{L^{2}\left(\Gamma_{k}\right)}-\left\langle u_{x}, v\right\rangle_{L^{2}(\Omega)} .
$$

Similarly, if we differentiate in the $y$-direction,

$$
\left\langle u, v_{y}\right\rangle_{L^{2}(\Omega)}=\sum_{k=w, e, s, n}\left\langle u, n_{y} v\right\rangle_{L^{2}\left(\Gamma_{k}\right)}-\left\langle u_{y}, v\right\rangle_{L^{2}(\Omega)} .
$$

We wish to replicate equation (6) and (7) in the discrete setting, using the inner products $\langle\cdot, \cdot\rangle_{\mathbf{P}_{\Omega}}$ and $\langle\cdot, \cdot\rangle_{\mathbf{P}_{\Gamma}}$. To this end, recall the metric relations

$$
J \xi_{x}=y_{\eta}, \quad J \xi_{y}=-x_{\eta}, \quad J \eta_{x}=-y_{\xi}, \quad J \eta_{y}=x_{\xi} .
$$

The relations (8) can be derived by inverting the Jacobian matrix $\frac{\partial(x, y)}{\partial(\xi, \eta)}$ and using the inverse function theorem to get $\frac{\partial(x, y)}{\partial(\xi, \eta)}{ }^{-1}=\frac{\partial(\xi, \eta)}{\partial(x, y)}$. From $(8)$ it follows that for a smooth function $u$ we have

$$
u_{x}=\xi_{x} u_{\xi}+\eta_{x} u_{\eta}=J^{-1}\left(y_{\eta} u_{\xi}-y_{\xi} u_{\eta}\right) .
$$

It is tempting to define the curvilinear operator using (9). However, the operator $\mathbf{J}^{-1}\left(\mathbf{Y}_{\eta} \mathbf{D}_{\xi}-\mathbf{Y}_{\xi} \mathbf{D}_{\eta}\right)$ will not mimic the integration by parts property 
(6). It will produce the correct boundary terms, but an incorrect volume term. In the continuous setting the following holds due to the product rule:

$$
\left(y_{\eta} u\right)_{\xi}-\left(y_{\xi} u\right)_{\eta}=y_{\eta \xi} u+y_{\eta} u_{\xi}-y_{\xi \eta} u-y_{\xi} u_{\eta}=y_{\eta} u_{\xi}-y_{\xi} u_{\eta} .
$$

Since the product rule does not hold in the discrete setting, the above relation must be somehow included in the SBP operator. This is known as a splitting technique [11. From (9) and (10) it follows that

$$
u_{x}=\frac{1}{2} J^{-1}\left(y_{\eta} u_{\xi}+\left(y_{\eta} u\right)_{\xi}-y_{\xi} u_{\eta}-\left(y_{\xi} u\right)_{\eta}\right) .
$$

Similarly,

$$
u_{y}=\frac{1}{2} J^{-1}\left(x_{\xi} u_{\eta}+\left(x_{\xi} u\right)_{\eta}-x_{\eta} u_{\xi}-\left(x_{\eta} u\right)_{\xi}\right) .
$$

The above expressions, when discretized, will mimic the integration by parts properties (6) and (7). We make the following definitions.

Definition 3. The matrices

$$
\mathbf{D}_{x}=\frac{1}{2} \mathbf{J}^{-1}\left(\mathbf{Y}_{\eta} \mathbf{D}_{\xi}+\mathbf{D}_{\xi} \mathbf{Y}_{\eta}-\mathbf{Y}_{\xi} \mathbf{D}_{\eta}-\mathbf{D}_{\eta} \mathbf{Y}_{\xi}\right)
$$

and

$$
\mathbf{D}_{y}=\frac{1}{2} \mathbf{J}^{-1}\left(\mathbf{X}_{\xi} \mathbf{D}_{\eta}+\mathbf{D}_{\eta} \mathbf{X}_{\xi}-\mathbf{X}_{\eta} \mathbf{D}_{\xi}-\mathbf{D}_{\xi} \mathbf{X}_{\eta}\right)
$$

are called SBP operators on $\Omega$ with respect to $\mathbf{P}$.

The operators $\mathbf{D}_{x}$ and $\mathbf{D}_{y}$ satisfy discrete analogues of equation (6) and (7) respectively. This fact will shortly be stated in the form of a proposition. However, a final component must first be translated to the discrete setting, namely the outward pointing unit normal $\left(n_{x}, n_{y}\right)$. Normals of the boundary can be expressed in terms of the gradients of $\xi$ and $\eta$. More precisely we have that

- $\nabla \xi$ is normal to the $(x, y)$-curves defined by $\xi=0$ and $\xi=1$ (i.e. $\Gamma_{w}$ and $\left.\Gamma_{e}\right)$.

- $\nabla \eta$ is normal to the $(x, y)$-curves defined by $\eta=0$ and $\eta=1$ (i.e. $\Gamma_{s}$ and $\left.\Gamma_{n}\right)$. 
Again, since the coordinate transform is typically not known, these gradients must be estimated. By using the metric relations (8), it follows that the normals can be approximated in terms of the partial derivatives $\mathbf{X}_{\xi}, \mathbf{X}_{\eta}, \mathbf{Y}_{\xi}, \mathbf{Y}_{\eta}$. Note for example that the vector $\left(-y_{\eta}, x_{\eta}\right) / \sqrt{x_{\eta}^{2}+y_{\eta}^{2}}$ is the outward pointing unit normal to the boundary $\{(x(0, \eta), y(0, \eta)): \eta \in[0,1]\}$. Similar expressions hold for the remaining boundaries, motivating the following definition.

Definition 4. The matrix pairs $\mathbf{N}^{k}=\left(\mathbf{N}_{x}^{k}, \mathbf{N}_{y}^{k}\right), k \in\{w, e, s, n\}$, where

$$
\begin{aligned}
& \mathbf{N}_{x}^{w}=-\left(E_{0} \otimes I_{\eta}\right) \mathbf{Y}_{\eta}\left(\mathbf{X}_{\eta}^{2}+\mathbf{Y}_{\eta}^{2}\right)^{-\frac{1}{2}} \\
& \mathbf{N}_{x}^{e}=\left(E_{M} \otimes I_{\eta}\right) \mathbf{Y}_{\eta}\left(\mathbf{X}_{\eta}^{2}+\mathbf{Y}_{\eta}^{2}\right)^{-\frac{1}{2}} \\
& \mathbf{N}_{x}^{s}=\left(I_{\xi} \otimes E_{0}\right) \mathbf{Y}_{\xi}\left(\mathbf{X}_{\xi}^{2}+\mathbf{Y}_{\xi}^{2}\right)^{-\frac{1}{2}} \\
& \mathbf{N}_{x}^{n}=-\left(I_{\xi} \otimes E_{N}\right) \mathbf{Y}_{\xi}\left(\mathbf{X}_{\xi}^{2}+\mathbf{Y}_{\xi}^{2}\right)^{-\frac{1}{2}}
\end{aligned}
$$

and

$$
\begin{aligned}
& \mathbf{N}_{y}^{w}=\left(E_{0} \otimes I_{\eta}\right) \mathbf{X}_{\eta}\left(\mathbf{X}_{\eta}^{2}+\mathbf{Y}_{\eta}^{2}\right)^{-\frac{1}{2}} \\
& \mathbf{N}_{y}^{e}=-\left(E_{M} \otimes I_{\eta}\right) \mathbf{X}_{\eta}\left(\mathbf{X}_{\eta}^{2}+\mathbf{Y}_{\eta}^{2}\right)^{-\frac{1}{2}} \\
& \mathbf{N}_{y}^{s}=-\left(I_{\xi} \otimes E_{0}\right) \mathbf{X}_{\xi}\left(\mathbf{X}_{\xi}^{2}+\mathbf{Y}_{\xi}^{2}\right)^{-\frac{1}{2}} \\
& \mathbf{N}_{y}^{n}=\left(I_{\xi} \otimes E_{N}\right) \mathbf{X}_{\xi}\left(\mathbf{X}_{\xi}^{2}+\mathbf{Y}_{\xi}^{2}\right)^{-\frac{1}{2}}
\end{aligned}
$$

are called the numerical boundary normals with respect to $\Omega_{h}$.

We may intuitively think of $\mathbf{N}_{x}^{k}$ and $\mathbf{N}_{y}^{k}$ as diagonal matrices with $n_{x}$ and $n_{y}$ evaluated at the boundary nodes in $\Gamma_{k}$.

The SBP property of $\mathbf{D}_{x}$ and $\mathbf{D}_{y}$ is now a straightforward consequence of the constructions above.

Proposition 1. For any $\mathbf{u}, \mathbf{v}$ on $\Omega_{h}$,

$$
\begin{aligned}
\left\langle\mathbf{u}, \mathbf{D}_{x} \mathbf{v}\right\rangle_{\mathbf{P}} & =\sum_{k=w, e, s, n}\left\langle\mathbf{u}, \mathbf{N}_{x}^{k} \mathbf{v}\right\rangle_{\mathbf{P}_{k}}-\left\langle\mathbf{D}_{x} \mathbf{u}, \mathbf{v}\right\rangle_{\mathbf{P}} \\
\left\langle\mathbf{u}, \mathbf{D}_{y} \mathbf{v}\right\rangle_{\mathbf{P}} & =\sum_{k=w, e, s, n}\left\langle\mathbf{u}, \mathbf{N}_{y}^{k} \mathbf{v}\right\rangle_{\mathbf{P}_{k}}-\left\langle\mathbf{D}_{y} \mathbf{u}, \mathbf{v}\right\rangle_{\mathbf{P}}
\end{aligned}
$$

We prove property $(13)$, the proof for $(14)$ is analogous. 
Proof. By Definition 1 and 3 we have

$$
\begin{aligned}
\left\langle\mathbf{u}, \mathbf{D}_{x} \mathbf{v}\right\rangle_{\mathbf{P}}= & \frac{1}{2} \mathbf{u}^{\top} \mathbf{P}_{\xi, \eta} \mathbf{J} \mathbf{J}^{-1}\left(\mathbf{Y}_{\eta} \mathbf{D}_{\xi}+\mathbf{D}_{\xi} \mathbf{Y}_{\eta}-\mathbf{Y}_{\xi} \mathbf{D}_{\eta}-\mathbf{D}_{\eta} \mathbf{Y}_{\xi}\right) \mathbf{v} \\
= & \frac{1}{2}\left\langle\mathbf{u}, \mathbf{Y}_{\eta} \mathbf{D}_{\xi} \mathbf{v}\right\rangle_{\mathbf{P}_{\xi, \eta}} \\
& +\frac{1}{2}\left\langle\mathbf{u}, \mathbf{D}_{\xi} \mathbf{Y}_{\eta} \mathbf{v}\right\rangle_{\mathbf{P}_{\xi, \eta}} \\
& -\frac{1}{2}\left\langle\mathbf{u}, \mathbf{Y}_{\xi} \mathbf{D}_{\eta} \mathbf{v}\right\rangle_{\mathbf{P}_{\xi, \eta}} \\
& -\frac{1}{2}\left\langle\mathbf{u}, \mathbf{D}_{\eta} \mathbf{Y}_{\xi} v\right\rangle_{\mathbf{P}_{\xi, \eta}}
\end{aligned}
$$

Here the inner product $\left\langle\mathbf{u}, \mathbf{D}_{x} \mathbf{v}\right\rangle_{\mathbf{P}}$ has been decomposed into four terms on which we will apply the SBP property (3). Since $\mathbf{Y}_{\eta}$ and $\mathbf{P}_{\xi, \eta}$ are diagonal we have $\left\langle\mathbf{u}, \mathbf{Y}_{\eta} \mathbf{D}_{\xi} \mathbf{v}\right\rangle_{\mathbf{P}_{\xi, \eta}}=\left\langle\mathbf{Y}_{\eta} \mathbf{u}, \mathbf{D}_{\xi} \mathbf{v}\right\rangle_{\mathbf{P}_{\xi, \eta}}$. By applying property (3) we get

$$
\left\langle\mathbf{Y}_{\eta} \mathbf{u}, \mathbf{D}_{\xi} \mathbf{v}\right\rangle_{\mathbf{P}_{\xi, \eta}}=\left\langle\mathbf{Y}_{\eta} \mathbf{u}, \mathbf{v}\right\rangle_{E_{M} \otimes P_{\eta}}-\left\langle\mathbf{Y}_{\eta} \mathbf{u}, \mathbf{v}\right\rangle_{E_{0} \otimes P_{\eta}}-\left\langle\mathbf{D}_{\xi} \mathbf{Y}_{\eta} \mathbf{u}, \mathbf{v}\right\rangle_{\mathbf{P}_{\xi, \eta}} .
$$

Furthermore, by Definition 2 and 4 we have

$$
\begin{aligned}
\left\langle\mathbf{Y}_{\eta} \mathbf{u}, \mathbf{v}\right\rangle_{E_{M} \otimes P_{\eta}} & =\left\langle\mathbf{u}, \mathbf{Y}_{\eta} \mathbf{v}\right\rangle_{E_{M} \otimes P_{\eta}} \\
& =\langle\mathbf{u},\left(\mathbf{X}_{\eta}^{2}+\mathbf{Y}_{\eta}^{2}\right)^{\frac{1}{2}} \underbrace{\mathbf{Y}_{\eta}\left(\mathbf{X}_{\eta}^{2}+\mathbf{Y}_{\eta}^{2}\right)^{-\frac{1}{2}}}_{\mathbf{N}_{x}^{e}} \mathbf{v}\rangle_{E_{M} \otimes P_{\eta}} \\
& =\left\langle\mathbf{u}, \mathbf{N}_{x}^{e} \mathbf{v}\right\rangle_{\mathbf{P}_{e}},
\end{aligned}
$$

and in the same way

$$
-\left\langle\mathbf{Y}_{\eta} \mathbf{u}, \mathbf{v}\right\rangle_{E_{0} \otimes P_{\eta}}=\left\langle\mathbf{u}, \mathbf{N}_{x}^{w} \mathbf{v}\right\rangle_{\mathbf{P}_{w}}
$$

Hence, the term 15 becomes

$$
\frac{1}{2}\left\langle\mathbf{u}, \mathbf{Y}_{\eta} \mathbf{D}_{\xi} \mathbf{v}\right\rangle_{\mathbf{P}_{\xi, \eta}}=\frac{1}{2}\left(\left\langle\mathbf{u}, \mathbf{N}_{x}^{e} \mathbf{v}\right\rangle_{\mathbf{P}_{e}}+\left\langle\mathbf{u}, \mathbf{N}_{x}^{w} \mathbf{v}\right\rangle_{\mathbf{P}_{w}}-\left\langle\mathbf{D}_{\xi} \mathbf{Y}_{\eta} \mathbf{u}, \mathbf{v}\right\rangle_{\mathbf{P}_{\xi}, \eta}\right) .
$$

Repeating the above reasoning for the remaining terms (16)-(18) yields

$$
\begin{aligned}
\frac{1}{2}\left\langle\mathbf{u}, \mathbf{D}_{\xi} \mathbf{Y}_{\eta} \mathbf{v}\right\rangle_{\mathbf{P}_{\xi, \eta}} & =\frac{1}{2}\left(\left\langle\mathbf{u}, \mathbf{N}_{x}^{e} \mathbf{v}\right\rangle_{\mathbf{P}_{e}}+\left\langle\mathbf{u}, \mathbf{N}_{x}^{w} \mathbf{v}\right\rangle_{\mathbf{P}_{w}}-\left\langle\mathbf{Y}_{\eta} \mathbf{D}_{\xi} \mathbf{u}, \mathbf{v}\right\rangle_{\mathbf{P}_{\xi}, \eta}\right) \\
-\frac{1}{2}\left\langle\mathbf{u}, \mathbf{Y}_{\xi} \mathbf{D}_{\eta} \mathbf{v}\right\rangle_{\mathbf{P}_{\xi, \eta}} & =\frac{1}{2}\left(\left\langle\mathbf{u}, \mathbf{N}_{x}^{n} \mathbf{v}\right\rangle_{\mathbf{P}_{n}}+\left\langle\mathbf{u}, \mathbf{N}_{x}^{s} \mathbf{v}\right\rangle_{\mathbf{P}_{s}}+\left\langle\mathbf{D}_{\eta} \mathbf{Y}_{\xi} \mathbf{u}, \mathbf{v}\right\rangle_{\mathbf{P}_{\xi}, \eta}\right) \\
-\frac{1}{2}\left\langle\mathbf{u}, \mathbf{D}_{\eta} \mathbf{Y}_{\xi} \mathbf{v}\right\rangle_{\mathbf{P}_{\xi, \eta}} & =\frac{1}{2}\left(\left\langle\mathbf{u}, \mathbf{N}_{x}^{n} \mathbf{v}\right\rangle_{\mathbf{P}_{n}}+\left\langle\mathbf{u}, \mathbf{N}_{x}^{s} \mathbf{v}\right\rangle_{\mathbf{P}_{s}}+\left\langle\mathbf{Y}_{\xi} \mathbf{D}_{\eta} \mathbf{u}, \mathbf{v}\right\rangle_{\mathbf{P}_{\xi}, \eta}\right)
\end{aligned}
$$


Finally, summing (21)-(24) we get,

$$
\begin{aligned}
\left\langle\mathbf{u}, \mathbf{D}_{x} \mathbf{v}\right\rangle_{\mathbf{P}}= & \sum_{k=w, e, s, n}\left\langle\mathbf{u}, \mathbf{N}_{x}^{k} \mathbf{v}\right\rangle_{\mathbf{P}_{k}} \\
& -\frac{1}{2}\left\langle\left(\mathbf{Y}_{\eta} \mathbf{D}_{\xi}+\mathbf{D}_{\xi} \mathbf{Y}_{\eta}-\mathbf{Y}_{\xi} \mathbf{D}_{\eta}-\mathbf{D}_{\eta} \mathbf{Y}_{\xi}\right) \mathbf{u}, \mathbf{v}\right\rangle_{\mathbf{P}_{\xi, \eta}} \\
= & \sum_{k=w, e, s, n}\left\langle\mathbf{u}, \mathbf{N}_{x}^{k} \mathbf{v}\right\rangle_{\mathbf{P}_{k}}-\left\langle\mathbf{D}_{x} \mathbf{u}, \mathbf{v}\right\rangle_{\mathbf{P}}
\end{aligned}
$$

A discrete version of Green's identity $\iint_{\Omega} u \Delta v d x d y=\int_{\partial \Omega} u \nabla v \cdot n d s-$ $\iint_{\Omega} u_{x} v_{x}+u_{y} v_{y} d x d y$ follows immediately from Proposition 1 .

Corollary 1. For any $\mathbf{u}, \mathbf{v}$ on $\Omega_{h}$,

$$
\langle\mathbf{u}, \Delta \mathbf{v}\rangle_{\mathbf{P}}=\sum_{k=w, e, s, n}\left\langle\mathbf{u}, \nabla \mathbf{v} \cdot \mathbf{N}^{k}\right\rangle_{\mathbf{P}_{k}}-\left\langle\mathbf{D}_{x} \mathbf{u}, \mathbf{D}_{x} \mathbf{v}\right\rangle_{\mathbf{P}}-\left\langle\mathbf{D}_{y} \mathbf{u}, \mathbf{D}_{y} \mathbf{v}\right\rangle_{\mathbf{P}}
$$

Remark 2. Curvilinear SBP operators are useful both for theoretical and practical purposes. Proposition 1 allows us to derive stability by mimicking the continuous energy method on the physical domain (which is typically where the boundary conditions are derived). Furthermore, the complexity of implementing curvilinear schemes is reduced since the grid transformation is built into the differentiation operators. The code for numerical differentiation on a curvilinear grid should be separate from any particular PDE solver, so that it can be debugged and tested in isolation. This reduces programming errors and produces managable solvers.

As an example of the simplification provided with the new formulation, consider discretizing a simple $2 \mathrm{~d}$ advection equation like $u_{t}+a u_{x}+b u_{y}=0$ on a curved domain. Using only standard SBP operators acting on square domains, the procedure is as follows:

1. Transform the PDE to the unit square using the chain rule: $u_{t}+\left(a \xi_{x}+\right.$ $\left.b \xi_{y}\right) u_{\xi}+\left(a \eta_{x}+b \eta_{y}\right) u_{\eta}=0$.

2. Apply metric relations: $J u_{t}+\left(a y_{\eta}-b x_{\eta}\right) u_{\xi}+\left(b x_{\xi}-a y_{\xi}\right) u_{\eta}=0$.

3. Note that the new equation is now a variable coefficient problem and that we must use a splitting technique to make our discretization stable: $J u_{t}+\frac{1}{2}\left(\left(a y_{\eta}-b x_{\eta}\right) u\right)_{\xi}+\frac{1}{2}\left(a y_{\eta}-b x_{\eta}\right) u_{\xi}+\frac{1}{2}\left(\left(b x_{\xi}-a y_{\xi}\right) u\right)_{\eta}+\frac{1}{2}\left(b x_{\xi}-\right.$ $\left.a y_{\xi}\right) u_{\eta}=0$. 
4. Multiply the equation from the previous step by $2 u$ and integrate in space. Integration by parts gives $\partial\|J u\|^{2} / \partial t+\int_{\partial I^{2}}\left(A n_{\xi}+B n_{\eta}\right) u^{2}=0$, where $A=a y_{\eta}-b x_{\eta}$ and $B=b x_{\xi}-a y_{\xi}$. From here we can derive boundary conditions that result in a well-posed problem.

5. Discretize with standard SBP operators acting on square domains: $\mathbf{J u}_{t}+\frac{1}{2} \mathbf{D}_{\xi}\left(a \mathbf{Y}_{\eta}-b \mathbf{X}_{\eta}\right) \mathbf{u}+\frac{1}{2}\left(a \mathbf{Y}_{\eta}-b \mathbf{X}_{\eta}\right) \mathbf{D}_{\xi} \mathbf{u}+\frac{1}{2} \mathbf{D}_{\eta}\left(b \mathbf{X}_{\xi}-a \mathbf{Y}_{\xi}\right) \mathbf{u}+$ $\frac{1}{2}\left(b \mathbf{X}_{\xi}-a \mathbf{Y}_{\xi}\right) \mathbf{D}_{\eta} \mathbf{u}=\mathrm{SAT}$, where the SAT is chosen according to the boundary conditions derived above.

6. Multiply the above equation by $2 \mathbf{u}^{\top} P_{\xi} \otimes P_{\eta}$ and repeatedly use the SBP properties of $\mathbf{D}_{\xi}$ and $\mathbf{D}_{\eta}$. This leads to $\partial\|\mathbf{u}\|_{\mathbf{J} P_{\xi} \otimes P_{\eta}} / \partial t+\langle\mathbf{u}, \mathbf{A u}\rangle_{\mathbf{P}_{e}}$ $\langle\mathbf{u}, \mathbf{A u}\rangle_{\mathbf{P}_{w}}+\langle\mathbf{u}, \mathbf{B u}\rangle_{\mathbf{P}_{n}}-\langle\mathbf{u}, \mathbf{B u}\rangle_{\mathbf{P}_{s}}=2\langle\mathbf{u}, \mathrm{SAT}\rangle_{P_{\xi} \otimes P_{\eta}}$, where $\mathbf{A}=$ $a \mathbf{Y}_{\eta}-b \mathbf{X}_{\eta}$ and $\mathbf{B}=a \mathbf{X}_{\xi}-b \mathbf{Y}_{\xi}$. From here we can derive a SAT term that results in a stable scheme.

Contrast this elaborate procedure with the following one, using curvilinear SBP operators:

1. Multiply the PDE by $2 u$ and integrate in space. Integration by parts gives $\partial\|u\|^{2} / \partial t+\int_{\partial \Omega} u^{2} \mathbf{a} \cdot \mathbf{n} d s=0$. From here we can derive boundary conditions that result in a well-posed problem.

2. Discretize the equation immediately: $\mathbf{u}_{t}+a \mathbf{D}_{x} \mathbf{u}+b \mathbf{D}_{y} \mathbf{u}=\mathrm{SAT}$.

3. Multiply the equation above by $2 \mathbf{u}^{\top} \mathbf{P}$ and use the SBP property from Proposition 1. This leads to $\partial\|\mathbf{u}\|_{\mathbf{P}}^{2} / \partial t+\sum_{k=w, e, s, n}\left\langle\mathbf{u}, \mathbf{a} \cdot \mathbf{N}^{k} \mathbf{u}\right\rangle_{\mathbf{P}_{k}}=$ $2\langle\mathbf{u}, \mathrm{SAT}\rangle$. From here we can derive a SAT term that results in a stable scheme.

Remark 3. The encapsulation reduces the complexity of three-dimensional (3D) discrete formulations to an even greater extent, since the metric algebra in the $3 D$ case increases dramatically.

\section{Projection operators}

Next we combine the curvilinear SBP operators with another reusability idea described in [6], where the authors construct general projection operators between non-conforming grids, with the aid of a so-called glue grid. 


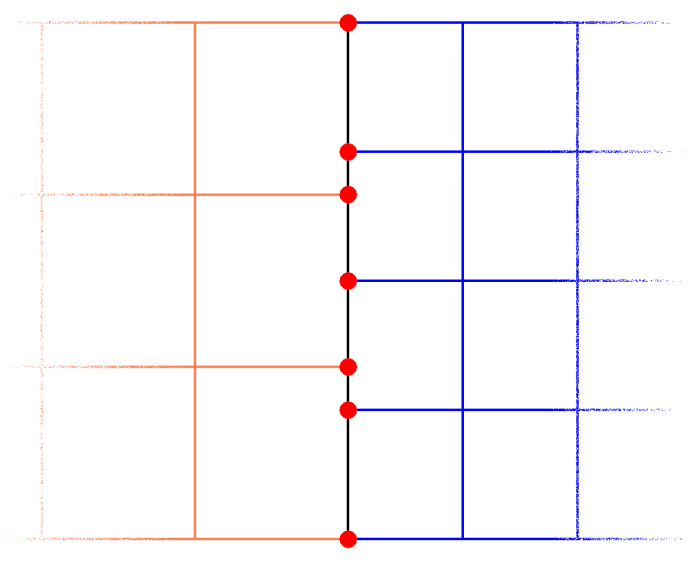

Figure 3: Intermediary glue grid formed by the union of the left and right interface grid points.

\subsection{Cartesian grids}

Let $\mathcal{I}=\Omega^{L} \cap \Omega^{R}$ be the interface at which the left and right subdomains meet (see Figure 11). Following [6] we construct a combined interface grid, called the glue grid, formed by ordering the union $\mathcal{I}_{h}=\left\{y_{j}^{L}\right\} \cup\left\{y_{j}^{R}\right\}$ of the left and right interface grid points (see Figure 3). Let the glue space $\mathcal{G}_{h} \subset L^{2}(\mathcal{I})$ be the space of piecewise polynomials of order $q_{i}-1$ on the subintervals defined by the grid points in $\mathcal{I}_{h}$, where $q_{i}$ is the interior order of the SBP operators. We denote by $\|\cdot\|$ and $\langle\cdot, \cdot\rangle$ the standard norm and inner product in $L^{2}(\mathcal{I})$, i.e. for $u, v \in L^{2}(\mathcal{I})$,

$$
\langle u, v\rangle:=\int_{\mathcal{I}} u v d y \quad \text { and } \quad\|u\|:=\sqrt{\int_{\mathcal{I}} u^{2} d y} .
$$

Given a basis $\left\{\psi_{i}\right\}_{i=1}^{K}$ in $\mathcal{G}_{h}$ (such a basis can be constructed for example by shifting Legendre polynomials as shown in [6]), the matrix $\mathbf{M}$ with elements $\int_{\mathcal{I}} \psi_{i} \psi_{j} d y$ defines an inner product on $\mathbb{R}^{K}$. If $\overline{\mathbf{u}}=\left(\bar{u}_{1}, \bar{u}_{2}, \ldots, \bar{u}_{K}\right)$ and $\overline{\mathbf{v}}=$ $\left(\bar{v}_{1}, \bar{v}_{2}, \ldots, \bar{v}_{K}\right)$ are vectors of weights corresponding to the functions $\bar{u}=$ $\sum \bar{u}_{i} \psi_{i}$ and $\bar{v}=\sum \bar{v}_{i} \psi_{i}$ in $\mathcal{G}_{h}$, then $\langle\bar{u}, \bar{v}\rangle=\langle\overline{\mathbf{u}}, \overline{\mathbf{v}}\rangle_{\mathbf{M}}$. Hence we will from now on think of $\mathcal{G}_{h}$ as the space $\mathbb{R}^{K}$ equipped with the inner product $\mathbf{M}$, and elements of $\mathcal{G}_{h}$ will always be denoted with an overbar. 
The projection operators [6]

$$
\begin{array}{ll}
\mathbf{F}_{\mathrm{lg}}: \mathbb{R}^{\left(N_{x}+1\right)\left(N_{y}+1\right)} \rightarrow \mathcal{G}_{h} & \mathbf{F}_{\mathrm{gl}}: \mathcal{G}_{h} \rightarrow \mathbb{R}^{\left(N_{x}+1\right)\left(N_{y}+1\right)} \\
\mathbf{F}_{\mathrm{rg}}: \mathbb{R}^{\left(M_{x}+1\right)\left(M_{y}+1\right)} \rightarrow \mathcal{G}_{h} & \mathbf{F}_{\mathrm{gr}}: \mathcal{G}_{h} \rightarrow \mathbb{R}^{\left(M_{x}+1\right)\left(M_{y}+1\right)}
\end{array}
$$

are such that if $\mathbf{u} \in \mathbb{R}^{\left(N_{x}+1\right)\left(N_{y}+1\right)}, \mathbf{v} \in \mathbb{R}^{\left(M_{x}+1\right)\left(M_{y}+1\right)}$, and $\overline{\mathbf{u}}, \overline{\mathbf{v}} \in \mathcal{G}_{h}$, then

$$
\begin{aligned}
\left\langle\mathbf{u}, \mathbf{F}_{\mathrm{gl}} \overline{\mathbf{v}}\right\rangle_{\mathbf{P}_{e}} & =\left\langle\mathbf{F}_{\mathrm{lg}} \mathbf{u}, \overline{\mathbf{v}}\right\rangle_{\mathbf{M}} \\
\left\langle\mathbf{v}, \mathbf{F}_{\mathrm{gr}} \overline{\mathbf{u}}\right\rangle_{\mathbf{H}_{w}} & =\left\langle\mathbf{F}_{\mathrm{rg}} \mathbf{v}, \overline{\mathbf{u}}\right\rangle_{\mathbf{M}},
\end{aligned}
$$

where $\mathbf{P}_{e}$ and $\mathbf{H}_{w}$ are the boundary quadratures from Section 3 . Here the subscripts indicate the direction of the projection (lg means the operator maps from the left grid to the glue grid, etc). The properties (26) and (27) are known as $\mathbf{P}_{e^{-}}$and $\mathbf{H}_{w^{-}}$compatibility and allow us to transfer grid representations of functions to piecewise polynomial representations in an inner product compatible manner. This is crucial for the stability analysis as it will allow us to penalize differences in the piecewise polynomial representations of the solutions, and to a large extent rely on already existing stability results from the conforming case.

The properties above can be formulated as the matrix equalities $\mathbf{P}_{e} \mathbf{F}_{\mathrm{gl}}=$ $\mathbf{F}_{\mathrm{lg}}^{\top} \mathbf{M}$ and $\mathbf{H}_{w} \mathbf{F}_{\mathrm{gr}}=\mathbf{F}_{\mathrm{rg}}^{\top} \mathbf{M}$ (this relation initially appeared in [8] under the term SBP preserving). These relations merely ensure inner product compatibility however, and have an infinite number of solutions. To ensure that the operators can be used to formulate consistent and useful schemes, accuracy constraints must be applied. For example, the operators $\mathbf{F}_{\mathrm{gl}}$ and $\mathbf{F}_{\mathrm{gr}}$ must map polynomials of order up to $q_{i}-1$ to their exact point values in the interior of the left and right interface grids. All the accuracy constraints, and the construction of the operators are given in [6], and not repeated here.

\subsection{Curvilinear grids}

Consider the two curved, quadrilateral domains $\Omega^{L}$ and $\Omega^{R}$ seen in Figure 4. Just like the standard SBP operators on rectangular grids were used to construct SBP operators on curvilinear grids, the standard projection operators from Section 5 can be used to construct projection operators on curved interfaces.

Let $\Omega_{h}^{L}$ and $\Omega_{h}^{R}$ be curvilinear grids in $\Omega^{L}$ and $\Omega^{R}$, with SBP quadratures $\mathbf{P}$ and $\mathbf{H}$ constructed as in Section 4. Furthermore, let $\mathbf{P}_{\mathcal{I}}$ and $\mathbf{H}_{\mathcal{I}}$ be SBP boundary quadratures on the interface $\mathcal{I}=\Omega^{L} \cap \Omega^{R}$. Then, from Section 4 


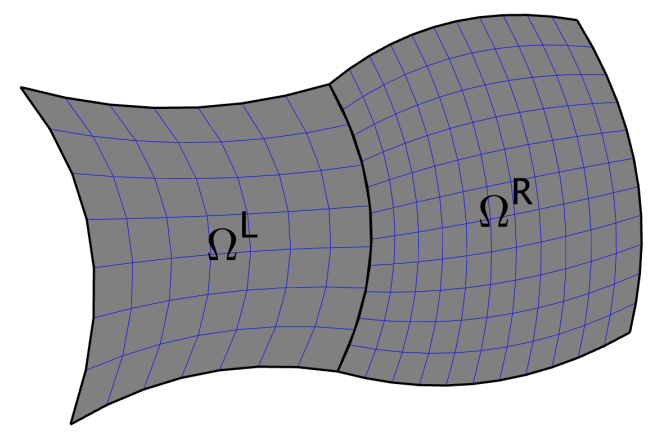

Figure 4: Two curvilinear, non-conforming grids.

$\mathbf{P}_{\mathcal{I}}=\left(E_{M} \otimes P_{\eta}\right) \mathbf{S}$ and $\mathbf{H}_{\mathcal{I}}=\left(E_{0} \otimes H_{\eta}\right) \mathbf{S}$, where $\mathbf{S}=\left(\mathbf{X}_{\eta}^{2}+\mathbf{Y}_{\eta}^{2}\right)^{\frac{1}{2}}$ (here $\mathbf{S}$ is taken from the left grid in $\mathbf{P}_{\mathcal{I}}$ and from the right grid in $\mathbf{H}_{\mathcal{I}}$ ). Now let $\mathbf{F}_{\mathrm{lg}}^{\eta}$, $\mathbf{F}_{\mathrm{gl}}^{\eta}, \mathbf{F}_{\mathrm{rg}}^{\eta}$ and $\mathbf{F}_{\mathrm{gr}}^{\eta}$ be standard $E_{M} \otimes P_{\eta^{-}}$and $E_{0} \otimes H_{\eta^{-}}$-compatible projection operators as in Section 5.

Using the above matrices we can define projection operators for the curved interface.

Definition 5. The matrices $\mathbf{F}_{l g}=\mathbf{F}_{l g}^{\eta} \mathbf{S}^{\frac{1}{2}}, \mathbf{F}_{g l}=\mathbf{S}^{-\frac{1}{2}} \mathbf{F}_{g l}^{\eta}, \mathbf{F}_{r g}=\mathbf{F}_{r g}^{\eta} \mathbf{S}^{\frac{1}{2}}$, and $\mathbf{F}_{g r}=\mathbf{S}^{-\frac{1}{2}} \mathbf{F}_{g r}^{\eta}$ are called curvilinear projection operators.

It is straightforward to prove that curvilinear projection operators preserve inner products in the same manner as the standard ones.

Proposition 2. The curvilinear projection operators $\mathbf{F}_{l g}, \mathbf{F}_{g l}, \mathbf{F}_{r g}$, and $\mathbf{F}_{g r}$

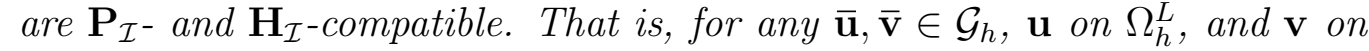
$\Omega_{h}^{R}$, we have

$$
\left\langle\mathbf{u}, \mathbf{F}_{g l} \overline{\mathbf{v}}\right\rangle_{\mathbf{P}_{\mathcal{I}}}=\left\langle\mathbf{F}_{l g} \mathbf{u}, \overline{\mathbf{v}}\right\rangle_{\mathbf{M}} \quad \text { and } \quad\left\langle\mathbf{v}, \mathbf{F}_{g r} \overline{\mathbf{u}}\right\rangle_{\mathbf{H}_{\mathcal{I}}}=\left\langle\mathbf{F}_{r g} \mathbf{v}, \overline{\mathbf{u}}\right\rangle_{\mathbf{M}}
$$

Proof.

$$
\begin{aligned}
\left\langle\mathbf{u}, \mathbf{F}_{\mathrm{gl}} \overline{\mathbf{v}}\right\rangle_{\mathbf{P}_{\mathcal{I}}} & =\left\langle\mathbf{u}, \mathbf{S}^{-\frac{1}{2}} \mathbf{F}_{\mathrm{gl}}^{\eta} \overline{\mathbf{v}}\right\rangle_{\mathbf{P}_{\mathcal{I}}}=\left\langle\mathbf{u}, \mathbf{S}^{\frac{1}{2}} \mathbf{F}_{\mathrm{gl}}^{\eta} \overline{\mathbf{v}}\right\rangle_{E_{M} \otimes P_{\eta}} \\
& =\left\langle\mathbf{S}^{\frac{1}{2}} \mathbf{u}, \mathbf{F}_{\mathrm{gl}}^{\eta} \overline{\mathbf{v}}\right\rangle_{E_{M} \otimes P_{\eta}}=\left\langle\mathbf{F}_{\mathrm{lg}}^{\eta} \mathbf{S}^{\frac{1}{2}} \mathbf{u}, \overline{\mathbf{v}}\right\rangle_{\mathbf{M}}=\left\langle\mathbf{F}_{\mathrm{lg}} \mathbf{u}, \overline{\mathbf{v}}\right\rangle_{\mathbf{M}}
\end{aligned}
$$

Here we have used that $\mathbf{S}$ is diagonal and the inner product preserving property (26). The proof for $\mathbf{F}_{\text {gr }}$ is analogous. 
Remark 4. The curvilinear projection and SBP operators encapsulate the whole discretization procedure. This is true both in terms of code and in terms of scheme design. Just like we can design schemes using standard SBP operators without really knowing how to construct the SBP operators (typically someone else will have done that for us already), we can design stable nonconforming curvilinear schemes using ready-made curvilinear SBP and projection operators directly. Note that these projection operators are identical to the ones constructed in [6], except for notation. In fact, in our numerical experiments we use the code supplied in [6] to construct the projection operators.

\section{Stability analysis for a non-conforming interface}

As an example of how the encapsulation theory above may be used to construct provably stable schemes in a concise manner we study an advectiondiffusion problem on a split domain with a non-conforming interface.

Let $\mathbf{a}=(a, b) \in \mathbb{R}^{2}, \epsilon>0, T>0$ and consider the following coupled advection-diffusion problem posed on the domain in Figure 4 .

$$
\begin{aligned}
u_{t}+\mathbf{a} \cdot \nabla u & =\epsilon \Delta u, \text { for }(x, y) \in \Omega^{L}, 0<t<T, \\
\left.u\right|_{t=0} & =f, \text { in } \Omega^{L}, \\
-\mathbf{a} \cdot \mathbf{n} u+\epsilon \nabla u \cdot \mathbf{n} & =g, \text { on } \partial \Omega_{\text {in }}, \\
\epsilon \nabla u \cdot \mathbf{n} & =h, \text { on } \partial \Omega_{\text {out }}, \\
v_{t}+\mathbf{a} \cdot \nabla v & =\epsilon \Delta v, \text { for }(x, y) \in \Omega^{R}, 0<t<T, \\
\left.v\right|_{t=0} & =f, \text { in } \Omega^{R}, \\
-\mathbf{a} \cdot \mathbf{n} v+\epsilon \nabla v \cdot \mathbf{n} & =g, \text { on } \partial \Omega_{\text {in }}, \\
\epsilon \nabla v \cdot \mathbf{n} & =h, \text { on } \partial \Omega_{\text {out }}, \\
u & =v, \text { on } \mathcal{I}, \\
\frac{\partial u}{\partial \mathbf{n}}+\frac{\partial v}{\partial \mathbf{n}} & =0, \text { on } \mathcal{I},
\end{aligned}
$$

were $\mathbf{n}$ is the outward pointing unit normal, $\partial \Omega_{\text {in }}=\partial \Omega \cap\{\mathbf{a} \cdot \mathbf{n}<0\}$, and $\partial \Omega_{\text {out }}=\partial \Omega \cap\{\mathbf{a} \cdot \mathbf{n} \geq 0\}$. 
The problem 29) can be shown to be well posed. It is discretized in space using the previously derived curvilinear SBP operators on the nonconforming grids. To impose the interface conditions we use SATs together with projection operators. For brevity we assume that the initial and external boundary conditions are implemented in a stable manner and in the following energy analysis we omit terms pertaining to the initial state and external boundaries.

Let $\partial \mathbf{u} / \partial \mathbf{n}=\mathbf{N}_{x}^{e} \mathbf{D}_{x} \mathbf{u}+\mathbf{N}_{y}^{e} \mathbf{D}_{y} \mathbf{u}, \partial \mathbf{v} / \partial \mathbf{n}=\mathbf{N}_{x}^{w} \mathbf{D}_{x} \mathbf{v}+\mathbf{N}_{y}^{w} \mathbf{D}_{y} \mathbf{v}$. The vectors $\partial \mathbf{u} / \partial \mathbf{n}$ and $\partial \mathbf{v} / \partial \mathbf{n}$ approximate the normal derivatives at the interface, with the normal pointing outward into the other domain.

Borrowing notation from [6], the semi-discrete scheme is formulated as

$$
\begin{aligned}
\mathbf{u}_{t}+\mathbf{a} \cdot \nabla \mathbf{u}-\epsilon \Delta \mathbf{u}= & \mathbf{P}^{-1} \mathbf{P}_{\mathcal{I}} \mathbf{F}_{\mathrm{gl}} \boldsymbol{\Sigma}_{L}^{I}(\overline{\mathbf{u}}-\overline{\mathbf{v}}) \\
& +\epsilon \mathbf{P}^{-1} \mathbf{P}_{\mathcal{I}} \mathbf{F}_{\mathrm{gl}} \boldsymbol{\Sigma}_{L}^{V}\left(\overline{\frac{\partial \mathbf{u}}{\partial \mathbf{n}}}+\frac{\bar{\partial}}{\partial \mathbf{n}}\right) \\
& +c_{L}^{I} \mathbf{P}^{-1} \mathbf{P}_{\mathcal{I}}\left(\mathbf{F}_{\mathrm{gl}}(\overline{\mathbf{a} \cdot \mathbf{N} e}) \overline{\mathbf{u}}-\left(\mathbf{a} \cdot \mathbf{N}^{e}\right) \mathbf{u}\right) \\
& +c_{L}^{V} \mathbf{P}^{-1} \mathbf{P}_{\mathcal{I}}\left(\mathbf{F}_{\mathrm{gl}} \frac{\overline{\mathbf{u}}}{\partial \mathbf{n}}-\frac{\partial \mathbf{u}}{\partial \mathbf{n}}\right) \\
\mathbf{v}_{t}+\mathbf{a} \cdot \nabla \mathbf{v}-\epsilon \Delta \mathbf{v}= & \mathbf{H}^{-1} \mathbf{H}_{\mathcal{I}} \mathbf{F}_{\mathrm{gr}} \boldsymbol{\Sigma}_{R}^{I}(\overline{\mathbf{v}}-\overline{\mathbf{u}}) \\
+ & \epsilon \mathbf{H}^{-1} \mathbf{H}_{\mathcal{I}} \mathbf{F}_{\mathrm{gr}} \boldsymbol{\Sigma}_{R}^{V}\left(\frac{\overline{\partial \mathbf{v}}}{\partial \mathbf{n}}+\frac{\partial \mathbf{u}}{\partial \mathbf{n}}\right) \\
& +c_{R}^{I} \mathbf{H}^{-1} \mathbf{H}_{\mathcal{I}}\left(\mathbf{F}_{\mathrm{gr}}(\overline{\mathbf{a} \cdot \mathbf{N} w}) \overline{\mathbf{v}}-\left(\mathbf{a} \cdot \mathbf{N}^{w}\right) \mathbf{v}\right) \\
& +c_{R}^{V} \mathbf{H}^{-1} \mathbf{H}_{\mathcal{I}}\left(\mathbf{F}_{\mathrm{gr}} \frac{\partial \mathbf{v}}{\partial \mathbf{n}}-\frac{\partial \mathbf{v}}{\partial \mathbf{n}}\right)
\end{aligned}
$$

where overbars indicate that a projection operator has been applied and transferred the grid function to its piecewise polynomial representation. The penalty terms (30a), 30b), 31a), 31b are nonconforming 2D generalizations of the penalty terms constructed in [1, while (30c), (30d), (31c), (31d) are correction terms similar to those used in [6]. In the stability analysis, the correction terms will replace interface terms originating from the left-hand side by their corresponding glue grid quantities.

$\boldsymbol{\Sigma}_{L, R}^{I, V}$ are diagonal matrices to be determined, and $\overline{\mathbf{a} \cdot \mathbf{N}^{e}}$ and $\overline{\mathbf{a} \cdot \mathbf{N}^{w}}$ are matrices on the glue grid approximating $\mathbf{a} \cdot \mathbf{N}^{e}$ and $\mathbf{a} \cdot \mathbf{N}^{w}$. They can 
be computed for example by solving for a diagonal matrix $\overline{\mathbf{a} \cdot \mathbf{N}}$ satisfying $\mathbf{F}_{\mathrm{gl}}(\overline{\mathbf{a} \cdot \mathbf{N}}) \mathbf{F}_{\mathrm{lg}}=\mathbf{a} \cdot \mathbf{N}^{e}$, and setting $\overline{\mathbf{a} \cdot \mathbf{N}^{e}}=\overline{\mathbf{a} \cdot \mathbf{N}}$ and $\overline{\mathbf{a} \cdot \mathbf{N}^{w}}=-\overline{\mathbf{a} \cdot \mathbf{N}}$. Diagonality of $\overline{\mathbf{a} \cdot \mathbf{N}}$ is needed for the formal stability proof, but numerical experiments show that also the intuitive non-diagonal choices $\overline{\mathbf{a} \cdot \mathbf{N}^{e}}=\mathbf{F}_{\lg }\left(\mathbf{a} \cdot \mathbf{N}^{e}\right) \mathbf{F}_{\mathrm{gl}}$ and $\overline{\mathbf{a} \cdot \mathbf{N}^{w}}=\mathbf{F}_{\mathrm{rg}}\left(\mathbf{a} \cdot \mathbf{N}^{w}\right) \mathbf{F}_{\mathrm{gr}}$ work in practice.

In the following proposition we use $\overline{\mathbf{a} \cdot \mathbf{N}^{e}}=\overline{\mathbf{a} \cdot \mathbf{N}}$ and $\overline{\mathbf{a} \cdot \mathbf{N}^{w}}=-\overline{\mathbf{a} \cdot \mathbf{N}}$, where $\overline{\mathbf{a} \cdot \mathbf{N}}$ is a diagonal matrix computed as explained above.

Proposition 3. The scheme (30) 31 is stable if

$$
\begin{gathered}
\boldsymbol{\Sigma}_{R}^{I}=\boldsymbol{\Sigma}_{L}^{I}-\overline{\mathbf{a} \cdot \mathbf{N}}, \quad \Sigma_{R}^{V}=-\left(\boldsymbol{\Sigma}_{L}^{V}+\mathbf{I}\right), \quad \boldsymbol{\Sigma}_{L}^{I} \leq \frac{\overline{\mathbf{a} \cdot \mathbf{N}}}{2}-\epsilon \frac{\left(\Sigma_{L}^{V}\right)^{2}+\left(\boldsymbol{\Sigma}_{R}^{V}\right)^{2}}{4 \xi} \\
c_{L}^{I}=-1 / 2, \quad c_{L}^{V}=\epsilon, \quad c_{R}^{I}=-1 / 2, \quad c_{R}^{V}=\epsilon,
\end{gathered}
$$

where $\xi$ is a positive number depending on the grid spacing, and $\boldsymbol{\Sigma}_{L, R}^{I, V}$ are diagonal matrices.

Proof. An expression for the rate of change in the energy of the solution is obtained by evaluating $2\langle\mathbf{u},(30)\rangle_{\mathbf{P}}$ and $2\langle\mathbf{v},(31)\rangle_{\mathbf{H}}$, i.e. by multiplying (30) and (31) by $2 \mathbf{u}^{\top} \mathbf{P}$ and $2 \mathbf{v}^{\top} \mathbf{H}$ respectively. Let us do this term-wise. Multiplying (30) by $2 \mathbf{u}^{\top} \mathbf{P}$ and applying Proposition 1 and 2 gives the following terms (omitting external boundary terms), originating from the left-hand side:

$$
\begin{aligned}
2\left\langle\mathbf{u}, \mathbf{u}_{t}\right\rangle_{\mathbf{P}} & =\frac{\partial}{\partial t}\|\mathbf{u}\|_{\mathbf{P}}^{2} \\
2\langle\mathbf{u}, \mathbf{a} \cdot \nabla \mathbf{u}\rangle_{\mathbf{P}} & =\left\langle\mathbf{u}, \mathbf{a} \cdot \mathbf{N}^{e} \mathbf{u}\right\rangle_{\mathbf{P}_{\mathcal{I}}} \\
2 \epsilon\langle\mathbf{u}, \Delta \mathbf{u}\rangle_{\mathbf{P}} & =2 \epsilon\left\langle\mathbf{u}, \frac{\partial \mathbf{u}}{\partial \mathbf{n}}\right\rangle_{\mathbf{P}_{\mathcal{I}}}-2 \epsilon\|\nabla \mathbf{u}\|_{\mathbf{P}}^{2} .
\end{aligned}
$$

And, from the right-hand side (here the numbering inside the inner products refer to the right hand side of equation (30)):

$$
\begin{aligned}
& 2\langle\mathbf{u},(30 \mathrm{a})\rangle_{\mathbf{P}}=2\left\langle\overline{\mathbf{u}}, \boldsymbol{\Sigma}_{L}^{I}(\overline{\mathbf{u}}-\overline{\mathbf{v}})\right\rangle_{\mathbf{M}} \\
& 2\langle\mathbf{u},(30 \mathrm{~b})\rangle_{\mathbf{P}}=2 \epsilon\left\langle\overline{\mathbf{u}}, \boldsymbol{\Sigma}_{L}^{V}\left(\frac{\overline{\partial \mathbf{u}}}{\partial \mathbf{n}}+\frac{\overline{\partial \mathbf{v}}}{\partial \mathbf{n}}\right)\right\rangle_{\mathbf{M}} \\
& 2\langle\mathbf{u}, 30 \mathrm{c})\rangle_{\mathbf{P}}=2 c_{L}^{I}\langle\overline{\mathbf{u}}, \overline{\mathbf{a} \cdot \mathbf{N}} \overline{\mathbf{u}}\rangle_{\mathbf{M}}-2 c_{L}^{I}\left\langle\mathbf{u}, \mathbf{a} \cdot \mathbf{N}^{e} \mathbf{u}\right\rangle_{\mathbf{P}_{\mathcal{I}}} \\
& 2\langle\mathbf{u},(30 \mathrm{~d})\rangle_{\mathbf{P}}=2 c_{L}^{V}\left\langle\overline{\mathbf{u}}, \frac{\overline{\partial \mathbf{u}}}{\partial \mathbf{n}}\right\rangle_{\mathbf{M}}-2 c_{L}^{V}\left\langle\mathbf{u}, \frac{\partial \mathbf{u}}{\partial \mathbf{n}}\right\rangle_{\mathbf{P}_{\mathcal{I}}} .
\end{aligned}
$$


Similarly, multiplying (31) by $2 \mathbf{v}^{\top} \mathbf{H}$ gives the following terms, originating from the left-hand side:

$$
\begin{aligned}
2\left\langle\mathbf{v}, \mathbf{v}_{t}\right\rangle_{\mathbf{H}} & =\frac{\partial}{\partial t}\|\mathbf{v}\|_{\mathbf{H}}^{2} \\
2\langle\mathbf{v}, \mathbf{a} \cdot \nabla \mathbf{v}\rangle_{\mathbf{H}} & =\left\langle\mathbf{v}, \mathbf{a} \cdot \mathbf{N}^{w} \mathbf{v}\right\rangle_{\mathbf{H}_{\mathcal{I}}} \\
2 \epsilon\langle\mathbf{v}, \Delta \mathbf{v}\rangle_{\mathbf{H}} & =2 \epsilon\left\langle\mathbf{v}, \frac{\partial \mathbf{v}}{\partial \mathbf{n}}\right\rangle_{\mathbf{H}_{\mathcal{I}}}-2 \epsilon\|\nabla \mathbf{v}\|_{\mathbf{H}}^{2} .
\end{aligned}
$$

And, from the right-hand side:

$$
\begin{aligned}
& 2\langle\mathbf{v}, 31 \mathrm{a})\rangle_{\mathbf{H}}=2\left\langle\overline{\mathbf{v}}, \boldsymbol{\Sigma}_{R}^{I}(\overline{\mathbf{v}}-\overline{\mathbf{u}})\right\rangle_{\mathbf{M}} \\
& 2\langle\mathbf{v}, 31 \mathrm{~b})\rangle_{\mathbf{H}}=2 \epsilon\left\langle\overline{\mathbf{v}}, \boldsymbol{\Sigma}_{R}^{V}\left(\frac{\overline{\partial \mathbf{v}}}{\partial \mathbf{n}}+\frac{\overline{\partial \mathbf{u}}}{\partial \mathbf{n}}\right)\right\rangle_{\mathbf{M}} \\
& 2\langle\mathbf{v}, 31 \mathrm{c})\rangle_{\mathbf{H}}=2 c_{R}^{I}\langle\overline{\mathbf{v}},-\overline{\mathbf{a} \cdot \mathbf{N}} \overline{\mathbf{v}}\rangle_{\mathbf{M}}-2 c_{R}^{I}\left\langle\mathbf{v}, \mathbf{a} \cdot \mathbf{N}^{w} \mathbf{v}\right\rangle_{\mathbf{H}_{\mathcal{I}}} \\
& 2\langle\mathbf{v}, 31 \mathrm{~d})\rangle_{\mathbf{H}}=2 c_{R}^{V}\left\langle\overline{\mathbf{v}}, \frac{\bar{\partial} \mathbf{v}}{\partial \mathbf{n}}\right\rangle_{\mathbf{M}}-2 c_{R}^{V}\left\langle\mathbf{v}, \frac{\partial \mathbf{v}}{\partial \mathbf{n}}\right\rangle_{\mathbf{H}_{\mathcal{I}}} .
\end{aligned}
$$

It follows that

$$
\begin{aligned}
\frac{\partial}{\partial t}\left(\|\mathbf{u}\|_{\mathbf{P}}^{2}+\right. & \left.\|\mathbf{v}\|_{\mathbf{H}}^{2}\right)= \\
& 2\left\langle\overline{\mathbf{u}},\left(\boldsymbol{\Sigma}_{L}^{I}-\overline{\mathbf{a} \cdot \mathbf{N}}\right) \overline{\mathbf{u}}\right\rangle_{\mathbf{M}}+2\left\langle\overline{\mathbf{v}},\left(\boldsymbol{\Sigma}_{R}^{I}+\overline{\mathbf{a} \cdot \mathbf{N}}\right) \overline{\mathbf{v}}\right\rangle_{\mathbf{M}} \\
+ & 2 \epsilon\left\langle\overline{\mathbf{u}},\left(\boldsymbol{\Sigma}_{L}^{V}+\mathbf{I}\right) \frac{\overline{\partial \mathbf{u}}}{\partial \mathbf{n}}\right\rangle_{\mathbf{M}}+2 \epsilon\left\langle\overline{\mathbf{v}},\left(\boldsymbol{\Sigma}_{R}^{V}+\mathbf{I}\right) \frac{\overline{\partial \mathbf{v}}}{\partial \mathbf{n}}\right\rangle_{\mathbf{M}} \\
- & 2\left\langle\overline{\mathbf{u}},\left(\boldsymbol{\Sigma}_{L}^{I}+\boldsymbol{\Sigma}_{R}^{I}\right) \overline{\mathbf{v}}\right\rangle_{\mathbf{M}}+2 \epsilon\left\langle\overline{\mathbf{u}}, \boldsymbol{\Sigma}_{L}^{V} \frac{\overline{\partial \mathbf{v}}}{\partial \mathbf{n}}\right\rangle_{\mathbf{M}}+2 \epsilon\left\langle\overline{\mathbf{v}}, \boldsymbol{\Sigma}_{R}^{V} \frac{\partial \overline{\mathbf{u}}}{\partial \mathbf{n}}\right\rangle_{\mathbf{M}} \\
- & 2 \epsilon\|\nabla \mathbf{u}\|_{\mathbf{P}}^{2}-2 \epsilon\|\nabla \mathbf{v}\|_{\mathbf{H}}^{2}
\end{aligned}
$$

The above is almost a quadratic form in $\overline{\mathbf{u}}, \overline{\mathbf{v}}, \overline{\partial \mathbf{u} / \partial \mathbf{n}}, \overline{\partial \mathbf{v} / \partial \mathbf{n}}$. In order to complete the quadratic form we must bound $\|\nabla \mathbf{u}\|_{\mathbf{P}}^{2}$ and $\|\nabla \mathbf{v}\|_{\mathbf{H}}^{2}$ from below in terms of $\|\overline{\partial \mathbf{u} / \partial \mathbf{n}}\|_{\mathbf{M}}^{2}$ and $\|\overline{\partial \mathbf{v} / \partial \mathbf{n}}\|_{\mathbf{M}}^{2}$. We do this for $\|\nabla \mathbf{u}\|_{\mathbf{P}}^{2}$ below, the proof for $\|\nabla \mathbf{v}\|_{\mathbf{H}}^{2}$ is analogous.

It is readily seen that there exists a constant $\alpha>0$ depending on the grid spacing such that $\|\nabla \mathbf{u}\|_{\mathbf{P}}^{2}=\left\|\mathbf{D}_{x} \mathbf{u}\right\|_{\mathbf{P}}^{2}+\left\|\mathbf{D}_{y} \mathbf{u}\right\|_{\mathbf{P}}^{2} \geq \alpha\left(\left\|\mathbf{D}_{x} \mathbf{u}\right\|_{\mathbf{P}_{\mathcal{I}}}^{2}+\left\|\mathbf{D}_{y} \mathbf{u}\right\|_{\mathbf{P}_{\mathcal{I}}}^{2}\right)$. Furthermore,

$$
\begin{aligned}
\left\|\mathbf{D}_{x} \mathbf{u}\right\|_{\mathbf{P}_{\mathcal{I}}}^{2}+\left\|\mathbf{D}_{y} \mathbf{u}\right\|_{\mathbf{P}_{\mathcal{I}}}^{2} & \geq\left\|\mathbf{N}_{x}^{e} \mathbf{D}_{x} \mathbf{u}\right\|_{\mathbf{P}_{\mathcal{I}}}^{2}+\left\|\mathbf{N}_{y}^{e} \mathbf{D}_{y} \mathbf{u}\right\|_{\mathbf{P}_{\mathcal{I}}}^{2} \\
& \geq \frac{1}{2}\left\|\mathbf{N}_{x}^{e} \mathbf{D}_{x} \mathbf{u}+\mathbf{N}_{y}^{e} \mathbf{D}_{y} \mathbf{u}\right\|_{\mathbf{P}_{\mathcal{I}}}^{2}=\frac{1}{2}\left\|\frac{\partial \mathbf{u}}{\partial \mathbf{n}}\right\|_{\mathbf{P}_{\mathcal{I}}}^{2} .
\end{aligned}
$$


Finally, using Proposition 2 ,

$$
\begin{aligned}
\| \overline{\frac{\partial \mathbf{u}}{\partial \mathbf{n}} \|_{\mathbf{M}}^{2}} & =\left\langle\frac{\overline{\partial \mathbf{u}}}{\partial \mathbf{n}}, \frac{\overline{\partial \mathbf{u}}}{\partial \mathbf{n}}\right\rangle_{\mathbf{M}}=\left\langle\frac{\partial \mathbf{u}}{\partial \mathbf{n}}, \mathbf{F}_{\mathrm{gl}} \frac{\overline{\partial \mathbf{u}}}{\partial \mathbf{n}}\right\rangle_{\mathbf{P}_{\mathcal{I}}}=\left\langle\frac{\partial \mathbf{u}}{\partial \mathbf{n}}, \mathbf{F}_{\mathrm{gl}} \mathbf{F}_{\lg } \frac{\partial \mathbf{u}}{\partial \mathbf{n}}\right\rangle_{\mathbf{P}_{\mathcal{I}}} \\
& \leq\left\|\mathbf{F}_{\mathrm{gl}} \mathbf{F}_{\lg }\right\|\left\|\frac{\partial \mathbf{u}}{\partial \mathbf{n}}\right\|_{\mathbf{P}_{\mathcal{I}}}^{2} .
\end{aligned}
$$

It follows that $\|\nabla \mathbf{u}\|_{\mathbf{P}}^{2} \geq \frac{\alpha}{2}\|\partial \mathbf{u} / \partial \mathbf{n}\|_{\mathbf{P}_{\mathcal{I}}}^{2} \geq \frac{\alpha}{2 \beta}\|\overline{\partial \mathbf{u} / \partial \mathbf{n}}\|_{\mathbf{M}}^{2}$, where $\beta=\left\|\mathbf{F}_{\mathrm{gl}} \mathbf{F}_{\text {lg }}\right\|$ (typically $\left\|\mathbf{F}_{\mathrm{gl}} \mathbf{F}_{\mathrm{lg}}\right\| \approx 1$ ). Hence there is a positive number $\xi$ depending on the grid resolutions and the projection operators such that $\xi\|\overline{\partial \mathbf{u} / \partial \mathbf{n}}\|_{\mathbf{M}}^{2} \leq$ $\|\nabla \mathbf{u}\|_{\mathbf{P}}^{2}$ and $\xi\|\overline{\partial \mathbf{v} / \partial \mathbf{n}}\|_{\mathbf{M}}^{2} \leq\|\nabla \mathbf{v}\|_{\mathbf{H}}^{2}$. Using this estimate in 48) gives

$$
\begin{aligned}
\frac{\partial}{\partial t}\left(\|\mathbf{u}\|_{\mathbf{P}}^{2}\right. & \left.+\|\mathbf{v}\|_{\mathbf{H}}^{2}\right) \leq \\
& 2\left\langle\overline{\mathbf{u}},\left(\boldsymbol{\Sigma}_{L}^{I}-\overline{\mathbf{a} \cdot \mathbf{N}}\right) \overline{\mathbf{u}}\right\rangle_{\mathbf{M}}+2\left\langle\overline{\mathbf{v}},\left(\boldsymbol{\Sigma}_{R}^{I}+\overline{\mathbf{a} \cdot \mathbf{N}}\right) \overline{\mathbf{v}}\right\rangle_{\mathbf{M}} \\
+ & 2 \epsilon\left\langle\overline{\mathbf{u}},\left(\boldsymbol{\Sigma}_{L}^{V}+\mathbf{I}\right) \frac{\overline{\partial \mathbf{u}}}{\partial \mathbf{n}}\right\rangle_{\mathbf{M}}+2 \epsilon\left\langle\overline{\mathbf{v}},\left(\boldsymbol{\Sigma}_{R}^{V}+\mathbf{I}\right) \frac{\overline{\partial \mathbf{v}}}{\partial \mathbf{n}}\right\rangle_{\mathbf{M}} \\
& -2\left\langle\overline{\mathbf{u}},\left(\boldsymbol{\Sigma}_{L}^{I}+\boldsymbol{\Sigma}_{R}^{I}\right) \overline{\mathbf{v}}\right\rangle_{\mathbf{M}}+2 \epsilon\left\langle\overline{\mathbf{u}}, \boldsymbol{\Sigma}_{L}^{V} \frac{\partial \overline{\mathbf{v}}}{\partial \mathbf{n}}\right\rangle_{\mathbf{M}}+2 \epsilon\left\langle\overline{\mathbf{v}}, \boldsymbol{\Sigma}_{R}^{V} \frac{\partial \overline{\mathbf{u}}}{\partial \mathbf{n}}\right\rangle_{\mathbf{M}} \\
& -2 \epsilon \xi\left\|\frac{\partial \mathbf{u}}{\partial \mathbf{n}}\right\|_{\mathbf{M}}^{2}-2 \epsilon \xi\left\|\frac{\partial \mathbf{v}}{\partial \mathbf{n}}\right\|_{\mathbf{M}}^{2} \\
= & \mathbf{w}^{\top} \mathbf{B} \mathbf{w},
\end{aligned}
$$

where $\mathbf{w}=\left(\overline{\mathbf{u}}^{\top}, \overline{\mathbf{v}}^{\top}, \overline{\partial \mathbf{u} / \partial \mathbf{n}}{ }^{\top}, \overline{\partial \mathbf{v} / \partial \mathbf{n}}{ }^{\top}\right)^{\top}$ and

$\mathbf{B}=\left[\begin{array}{ccccc}\mathbf{M} & & & \\ & \mathbf{M} & & \\ & & \mathbf{M} & \\ & & & \mathbf{M}\end{array}\right]\left[\begin{array}{cccc}2 \boldsymbol{\Sigma}_{L}^{I}-\overline{\mathbf{a} \cdot \mathbf{N}} & -\left(\boldsymbol{\Sigma}_{L}^{I}+\boldsymbol{\Sigma}_{R}^{I}\right) & \epsilon\left(\boldsymbol{\Sigma}_{L}^{V}+\mathbf{I}\right) & \epsilon \boldsymbol{\Sigma}_{L}^{V} \\ -\left(\boldsymbol{\Sigma}_{L}^{I}+\boldsymbol{\Sigma}_{R}^{I}\right) & 2 \boldsymbol{\Sigma}_{R}^{I}+\overline{\mathbf{a} \cdot \mathbf{N}} & \epsilon \boldsymbol{\Sigma}_{R}^{V} & \epsilon\left(\boldsymbol{\Sigma}_{R}^{V}+\mathbf{I}\right) \\ \epsilon\left(\boldsymbol{\Sigma}_{L}^{V}+\mathbf{I}\right) & \epsilon \boldsymbol{\Sigma}_{R}^{V} & -2 \epsilon \xi \mathbf{I} & 0 \\ \epsilon \boldsymbol{\Sigma}_{L}^{V} & \epsilon\left(\boldsymbol{\Sigma}_{R}^{V}+\mathbf{I}\right) & 0 & -2 \epsilon \xi \mathbf{I}\end{array}\right]$.

Since $\mathbf{M}$ can be chosen diagonal by using orthogonal basis functions in the glue space, all blocks in $\mathbf{B}$ are diagonal if $\overline{\mathbf{a} \cdot \mathbf{N}}$ is diagonal. It follows from [1] that $\mathbf{B}$ is negative semi-definite if 32 holds.

Remark 5. Note that unlike the stability analysis in [6], the above proof does not involve any metric coefficients or grid transformations explicitly. Instead we rely on the fact that our operators act immediately on the curved grid. The grid transformation part has been entirely abstracted away. 


\section{Numerical results}

In this section we discuss numerical stability results, freestream preservation and potential advantages of non-conforming grids in large scale fluid simulations.

\subsection{Convergence and stability}

We test our scheme with the parameters $a=1, b=0.5, \epsilon=0.01$, by pulling data from the manufactured solution $\cos (10 x+10 y+t)$. The left and right subdomains are discretized using two non-conforming blocks (or elements) as in Figure 4. Solutions and $L^{2}$-errors in space are computed at time $T=1$. The first discretization uses a $24 \times 24$-grid in the left subdomain and a $48 \times 48$-grid in the right subdomain. Thereafter the subdomain resolutions are increased and a convergence rate is computed as $\left(\log \left(e_{\text {coarse }}\right)-\log \left(e_{\text {fine }}\right)\right) /\left(\log \left(h_{\text {coarse }}\right)-\log \left(h_{\text {fine }}\right)\right)$. The errors $e_{\text {coarse }}$ and $e_{\text {fine }}$ are given by $\sqrt{\left\|\mathbf{u}-\mathbf{u}_{\text {exact }}\right\|_{\mathbf{P}}^{2}+\left\|\mathbf{v}-\mathbf{v}_{\text {exact }}\right\|_{\mathbf{H}}^{2}}$, where $\mathbf{u}_{\text {exact }}$ and $\mathbf{v}_{\text {exact }}$ are the exact solutions evaluated at the grid nodes. The notation $\operatorname{SBP}(2 p, p)$ means that the operator is of order $2 p$ for interior nodes and $p$ for boundary nodes. The scheme $30-(31)$ using only $\operatorname{SBP}(2 p, p)$ operators is $p+1$ order accurate (we have used first derivative operators twice to approximate the second derivative). Numerical results for various order $\operatorname{SBP}(2 p, p)$ operators are listed in Table 7.1, and matches the design order of the scheme.

We further illustrate the stability of the scheme by studying energy preservation in the case of pure advection $(\epsilon=0, \mathbf{a}=(1.0,0.1))$, using the domain in Figure 4 with a $30 \times 30$ grid on the left and a $40 \times 40$ grid on the right. We use a Gauss bell as initial data and let it advect across the interface. The energy of the solution is computed at all time steps and shown in Figure 5.

\subsection{Freestream preservation}

Exact freestream preservation means that the time derivative $\mathbf{u}_{t}$ of the discretization (30)-31) vanishes for constant initial and boundary data. It is important to note that this property cannot be guaranteed for arbitrary curvilinear grids using the projection operators discussed in Section 5 . The projection operators are exact for polynomials up to order $q_{b}-1$, where $q_{b}$ is the boundary order of accuracy of the SBP operators (see [6]). Hence, as long as the interface is described by such a polynomial, the scheme will be exactly freestream preserving (the metric terms will be mapped exactly into the glue space). However, if the interface does not have this property, the 


\begin{tabular}{|c|c|c|}
\hline \multicolumn{3}{|c|}{$\operatorname{SBP}(2,1)$} \\
\hline res & error & rate \\
\hline $24 / 48$ & $2.26 \mathrm{e}-02$ & \\
\hline $48 / 96$ & $5.45 \mathrm{e}-03$ & 1.99 \\
\hline $96 / 198$ & $1.33 \mathrm{e}-03$ & 2.00 \\
\hline $198 / 396$ & $3.10 \mathrm{e}-04$ & 2.00 \\
\hline
\end{tabular}

\begin{tabular}{|r|c|c|}
\multicolumn{4}{|c}{$\operatorname{SBP}(4,2)$} \\
\hline res & error & rate \\
\hline $24 / 48$ & $9.01 \mathrm{e}-03$ & \\
$48 / 96$ & $1.04 \mathrm{e}-03$ & 3.02 \\
$96 / 198$ & $1.23 \mathrm{e}-04$ & 3.04 \\
$198 / 396$ & $1.35 \mathrm{e}-05$ & 3.03 \\
\hline
\end{tabular}

\begin{tabular}{|r|c|c|}
\multicolumn{4}{c}{$\operatorname{SBP}(6,3)$} \\
\hline res & error & rate \\
\hline $24 / 48$ & $1.25 \mathrm{e}-02$ & \\
$48 / 96$ & $8.64 \mathrm{e}-04$ & 3.73 \\
$96 / 198$ & $7.68 \mathrm{e}-05$ & 3.44 \\
$198 / 396$ & $4.93 \mathrm{e}-06$ & 3.77 \\
\hline
\end{tabular}

\begin{tabular}{|r|c|c|}
\multicolumn{4}{|c}{$\operatorname{SBP}(8,4)$} \\
\hline res & error & rate \\
\hline $24 / 48$ & $7.28 \mathrm{e}-03$ & \\
$48 / 96$ & $4.15 \mathrm{e}-04$ & 4.01 \\
$96 / 198$ & $1.47 \mathrm{e}-05$ & 4.75 \\
$198 / 396$ & $3.88 \mathrm{e}-07$ & 4.98 \\
\hline
\end{tabular}

Table 1: $L^{2}$ errors at time $T=1$ and convergence rates for different SBP operators on the domain in Figure 4. The res column displays the resolution in the format $N / M$, meaning the left subdomain uses an $N \times N$-grid and the right subdomain uses an $M \times M$-grid.
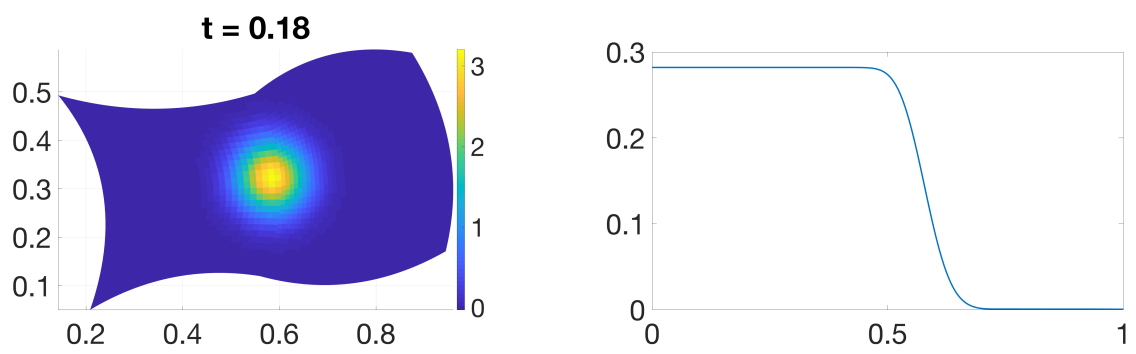

Figure 5: Left: Plot of Gauss bell as it advects across a non-conforming interface. Right: Plot of energy over time. The energy of the solution is preserved until the Gauss bell exits the domain. 

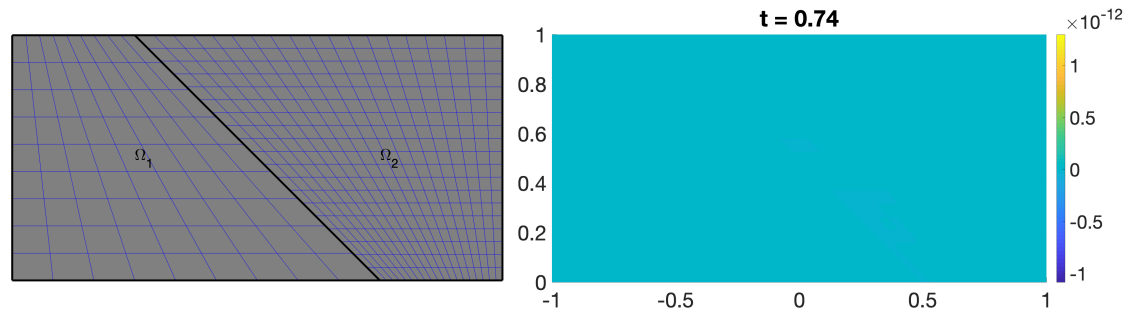

Figure 6: Left: Low order polynomial non-conforming interface. The grids are of size $10 \times 10$ and $20 \times 20$ respectively. Right: Error plot (deviation from 1). Freestream preservation up to machine precision since the projection operators can map the metric terms exactly.

projections of the metric terms will not be exact, i.e. the projection from the left domain into the glue space will not be identical to the projection from the right domain into the glue space (although the difference will tend to zero with grid refinement).

To illustrate this phenomenon, consider first the simple diagonal interface seen in Figure 6. The left grid consists of $10 \times 10$ gridpoints and the right grid consits of $20 \times 20$ gridpoints. We run a simulation with the parameters $\mathbf{a}=(1.0,0.1), \epsilon=0.01$, using $\operatorname{SBP}(4,2)$ operators and constant initial and boundary data. This results in freestream preservation. Contrast this with the same simulation on a grid with an interface that is not exactly represented in the glue space. Such a simulation can be seen in Figure 7 , where the first grid is again $10 \times 10$ on the left and $20 \times 20$ on the right (we then grid refine by doubling the number of gridpoints in each direction). Here the freestream state is not preserved, but the error tends to zero with grid refinement. Note also that the largest freestream error is introduced where the gradients are large.

Finally we stress that the lack of freestream preservation has nothing to do with the encapsulation procedure. It is only due to the lack of accuracy in the projection procedure. For conforming grids, freestream preservation is attained. This can be seen in Figure 8.

\subsection{Airfoil simulation}

To illustrate the potential efficiency gains associated with non-conforming interfaces, and the applicability of our encapsulation procedure, consider the domain shown in Figure 9. In a flow simulation we might be interested in the temperature distribution in the fluid as it hits an airfoil. In such a case, the 

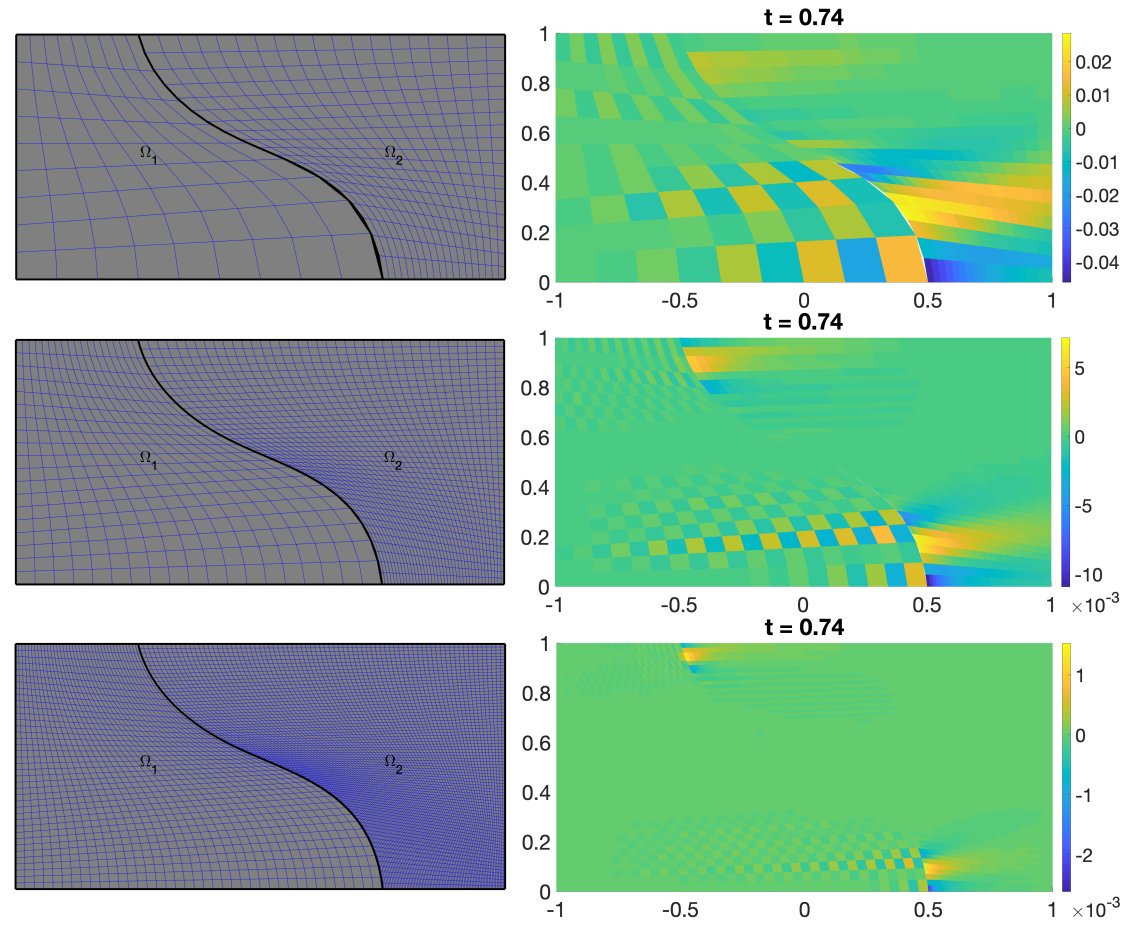

Figure 7: Left: Non-polynomial non-conforming interface. The grid sizes are, from top to bottom: $(10 \times 10,20 \times 20),(20 \times 20,40 \times 40),(40 \times 40,80 \times 80)$. Right: Error plots (deviation from 1). No exact freestream preservation since the projection operators do not map the metric terms exactly. The error vanishes with grid refinement.
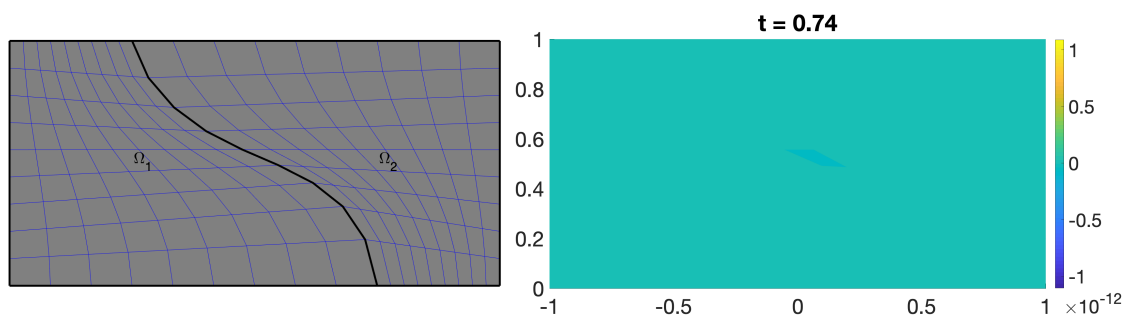

Figure 8: Left: Non-polynomial conforming interface. Both grids are of size $10 \times 10$. Right: Error plot (deviation from 1). Freestream preservation up to machine precision since for a conforming interface our penalty terms do not involve any interpolation of the metric terms. 


\begin{tabular}{|c|c|c|c|c|}
\hline res & conf. runtime & non-conf. runtime & conf. error & non-conf. error \\
\hline 31 & $6.9 \mathrm{~s}$ & $6.9 \mathrm{~s}$ & 0.0862 & 0.0862 \\
61 & $16.2 \mathrm{~s}$ & $9.4 \mathrm{~s}$ & 0.0209 & 0.0209 \\
121 & $63.7 \mathrm{~s}$ & $22.3 \mathrm{~s}$ & 0.0048 & 0.0049 \\
\hline
\end{tabular}

Table 2: Execution times and errors of the steady state solution (seen in Figure 10 with global, conforming grid refinement versus local, non-conforming grid refinement. The rescolumn shows the resolution of the grid in $\Omega_{1}$. In the non-conforming case, the $\Omega_{2}$ and $\Omega_{3}$-grids are held fixed at $31 \times 31$, whereas in the conforming case they have the same resolution as the $\Omega_{1}$-grid.

computational domain needs to be large so that the area of interest is not disturbed by reflections from nearby external boundaries. On the other hand it needs to be sufficiently fine in regions of interest. Non-conforming interfaces allow us to propagate the temperature field from the fine grid straight into a coarse grid when we no longer require high accuracy, see Figure 9, thus reducing the computational load for parts of the domain whose main purpose is to carry the solution toward the far field boundary.

Figure 10 shows a reference solution to the advection-diffusion problem with parameters $\mathbf{a}=(1,0)$ and $\epsilon=0.1$. A homogeneous Dirichlet condition is enforced on the airfoil. At inflow and outflow boundaries, the boundary conditions in 29) are used, with constant inflow data and zero outflow data. The steady state reference solution uses $241 \times 241$ grids in each of the subdomains in Figure 9. We compute errors inside $\Omega_{1}$ with respect to the reference solution, and execution times for conforming and non-conforming grid refinement. By conforming grid refinement we mean that all three grids are refined together so that they conform at the interface. The non-conforming refinement is done by holding the grids in $\Omega_{2}$ and $\Omega_{3}$ fixed, while refining in $\Omega_{1}$ only. Since we are only interested in the solution inside $\Omega_{1}$ we will lower the computational cost by grid refining inside $\Omega_{1}$ independently of $\Omega_{2}$ and $\Omega_{3}$. Indeed, local grid refining inside $\Omega_{1}$ produces the same errors as global refinement, but at reduced execution times, as seen in Table 2. The simulations were run on a $2.7 \mathrm{GHz}$ Intel Core i 5 with 2 cores.

\section{Conclusions}

By regarding grid transformations as part of operator construction we were able to construct SBP operators acting directly on curvilinear grids. We have shown how to construct non-conforming couplings using curvilinear 


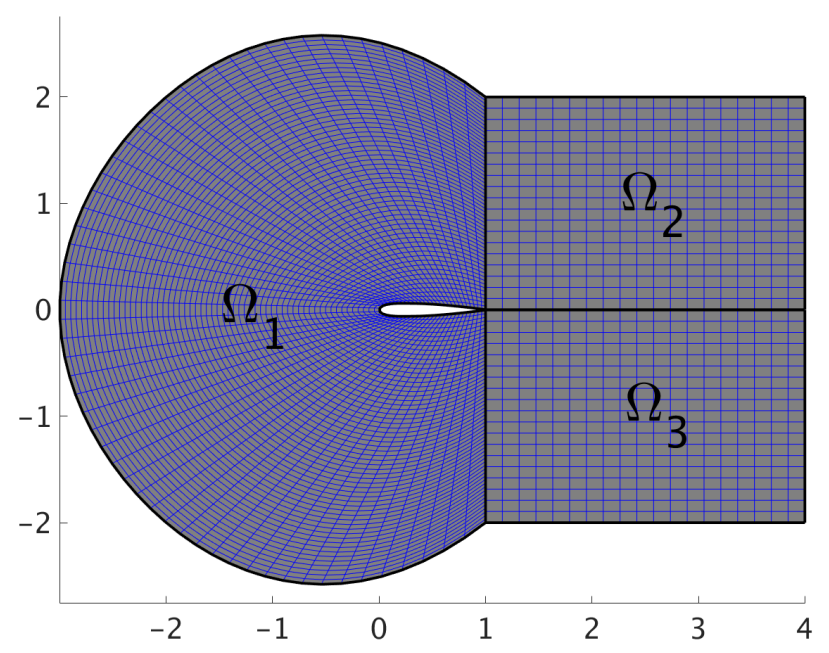

Figure 9: A non-conforming airfoil grid.

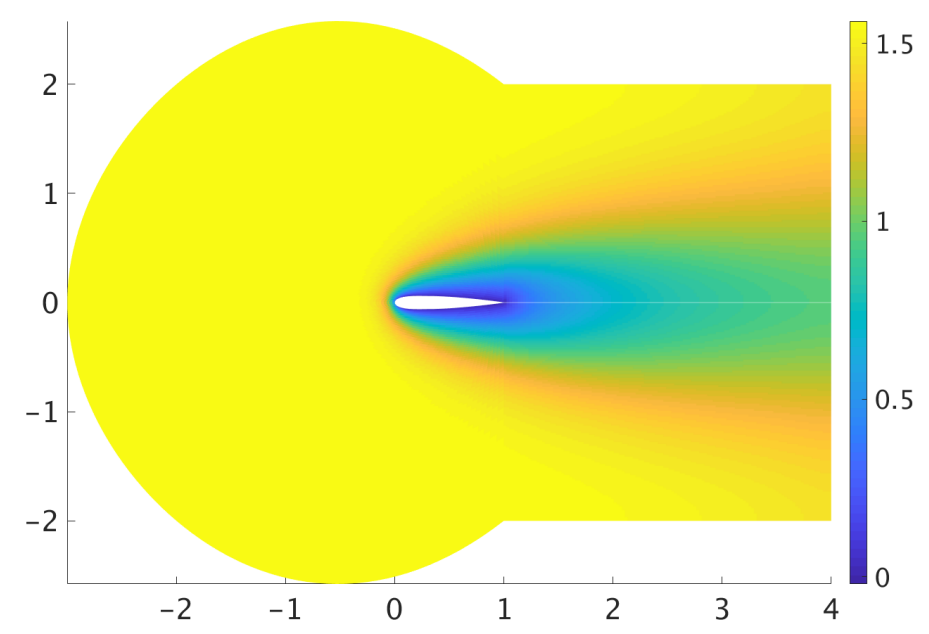

Figure 10: Temperature distribution around an airfoil. 
SBP operators and projection operators, and how to reduce the stability analysis to the known conforming case. This theoretical development enables the production of simple encapsulated, reusable, and easy to manage codes.

Our numerical experiments showed no reduction in convergence rates compared to the conforming case. We also illustrated the energy and freestream preserving properties of the scheme. Furthermore we showed that execution times can be reduced compared to conforming grid refinement, since the lack of conformity requirements allowed us to adjust the grid resolution in one block independently of its neighbors.

\section{References}

[1] M. H. Carpenter, J. Nordström, and D. Gottlieb. A stable and conservative interface treatment of arbitrary spatial accuracy. Journal of Computational Physics, 148:341-365, 1999.

[2] D. C. D. R. Fernández, J. E. Hicken, and D. W. Zingg. Review of summation-by-parts operators with simultaneous approximation terms for the numerical solution of partial differential equations. Computers E3 Fluids, 95:171-196, 2014.

[3] D.C.D.R. Fernández, J.E. Hicken, and D.W. Zingg. Simultaneous approximation terms for multi-dimensional summation-by-parts operators. Journal of Scientific Computing, 75(1):83-110, 2018.

[4] G.J. Gassner. A skew-symmetric discontinuous Galerkin spectral element discretization and its relation to SBP-SAT finite difference methods. SIAM Journal on Scientific Computing, 35(3):A1233-A1253, 2013.

[5] Jason E Hicken, David C Del Rey Fernández, and David W Zingg. Multidimensional summation-by-parts operators: General theory and application to simplex elements. SIAM Journal on Scientific Computing, 38(4):A1935-A1958, 2016.

[6] J. E. Kozdon and L. C. Wilcox. Stable coupling of nonconforming, highorder finite difference methods. SIAM Journal on Scientific Computing, 38:A923-A952, 2016.

[7] T. Lundquist, A. Malan, and J. Nordström. A hybrid framework for coupling arbitrary summation-by-parts schemes on general meshes. Journal of Computational Physics, 362:49-68, 2018. 
[8] K. Mattsson and M. H. Carpenter. Stable and accurate interpolation operators for high-order multiblock finite difference methods. SIAM Journal on Scientific Computing, 32:2298-2320, 2010.

[9] K. Mattsson, F. Ham, and G. Iaccarino. Stable and accurate wavepropagation in discontinuous media. Journal of Computational Physics, 227(19):8743-8767, 2008.

[10] Jan Nordström and Mark H Carpenter. High-order finite difference methods, multidimensional linear problems, and curvilinear coordinates. Journal of Computational Physics, 173(1):149-174, 2001.

[11] J. Nordström. Conservative finite difference formulations, variable coefficients, energy estimates and artificial dissipation. Journal of Scientific Computing, 29(3):375-404, 2006.

[12] J. Nordström, K. Forsberg, C. Adamsson, and P. Eliasson. Finite volume methods, unstructured meshes and strict stability for hyperbolic problems. Applied Numerical Mathematics, 45(4):453-473, 2003.

[13] N Anders Petersson and Björn Sjögreen. Wave propagation in anisotropic elastic materials and curvilinear coordinates using a summation-by-parts finite difference method. Journal of Computational Physics, 299:820-841, 2015.

[14] H. Ranocha, P. Öffner, and T. Sonar. Summation-by-parts operators for correction procedure via reconstruction. Journal of Computational Physics, 311:299-328, 2016.

[15] Magnus Svärd, Mark H Carpenter, and Jan Nordström. A stable highorder finite difference scheme for the compressible navier-stokes equations, far-field boundary conditions. Journal of Computational Physics, 225(1):1020-1038, 2007.

[16] Magnus Svärd and Jan Nordström. A stable high-order finite difference scheme for the compressible navier-stokes equations: no-slip wall boundary conditions. Journal of Computational Physics, 227(10):4805-4824, 2008. 
[17] M. Svärd and J. Nordström. Review of summation-by-parts schemes for initial-boundary-value problems. Journal of Computational Physics, 268:17-38, 2014. 